Pacific

Journal of

Mathematics

\title{
CHARACTERIZATION OF A GENERALIZED SHANKS SEQUENCE
}

Roger D. Patterson, Alfred J. Van der Poorten AND Hugh C. Williams 


\title{
CHARACTERIZATION OF A GENERALIZED SHANKS SEQUENCE
}

\author{
Roger D. Patterson, Alfred J. VAn der Poorten \\ AND Hugh C. WiLliams
}

\begin{abstract}
We consider generalizations of Shanks' sequence of quadratic fields $\mathbb{Q}\left(\sqrt{S_{n}}\right)$ where $S_{n}=\left(2^{n}+1\right)^{2}+2^{n+2}$. Quadratic fields of this type are of interest because it is possible to explicitly determine the fundamental unit. If a sequence of quadratic fields given by $D_{n}=A^{2} x^{2 n}+B x^{n}+C^{2}$ satisfies certain conditions (notably that the regulator is of order $\Theta\left(n^{2}\right)$ ), then we determine the exact form such a sequence must take.
\end{abstract}

\section{History of creepers}

We will be interested in simple continued fractions, which we denote by $\alpha=$ [ $\left.a_{0}, a_{1}, a_{1}, a_{2}, \ldots\right]$ ]; the $a_{i}$ are called the partial quotients of $\alpha$. It is well-known that a continued fraction expansion is periodic if and only if it is the expansion of a real quadratic irrational. We denote the period length of a real quadratic irrational $\alpha$ by $\operatorname{lp}(\alpha)$.

For real quadratic fields, it is expected that the class number will usually be small; see [Cohen and Lenstra 1984]. By the correspondence between ideals and continued fractions this is equivalent to the continued fraction expansion of $\sqrt{D}$ being long, generally of length about $\sqrt{D}$. Thus, examples of short expansions of $\sqrt{D}$ should be considered as unusual and worthy of interest.

It is easy to find sequences of integers $D_{i}$ such that $\sqrt{D_{i}}$ has a bounded period length. Many results have been determined for such families and we refer the reader to [Perron 1950; van der Poorten and Williams 1999; Schinzel 1960; 1961].

Shanks [1969] examined the class numbers of quadratic fields with discriminants given by $n^{2}-2^{2 k+1}$. He noticed that for the family $S_{n}=\left(2^{n}+3\right)^{2}-8$, the class number of $S_{n}$ grows infinitely large. This sequence of fields is known as Shanks' sequence. It happens that Shanks' sequence is just a special case of an earlier

MSC2000: primary 11R11, 11R27; secondary 11G20.

Keywords: periodic continued fraction, quadratic order, units.

Van der Poorten was supported in part by a grant from the Australian Research Council. 
example given in [Nyberg 1949]:

$$
D_{n}=\left(x^{n}+(x \pm 1) / 2\right)^{2} \mp x^{n} .
$$

The ring of algebraic integers of a quadratic number field $K=\mathbb{Q}(\sqrt{D})$, denoted by $O_{K}$, is equal to $\mathbb{Z}\left[\omega^{\prime}\right]$, where $d_{K}$ is the squarefree kernel of $D$ and

$$
\omega^{\prime}=\left\{\begin{array}{lll}
\sqrt{d_{K}} & \text { if } d_{K} \neq 1 & (\bmod 4), \\
\left(1+\sqrt{d_{K}}\right) / 2 & \text { if } d_{K} \equiv 1 & (\bmod 4) .
\end{array}\right.
$$

The discriminant, $D_{K}$, of $\mathscr{O}_{K}$ is equal to $d_{K}$ if $d_{K}$ is congruent to 1 modulo 4 and $4 d_{K}$ otherwise. An order 0 of $K$ is defined to be a subring of $K$, containing 1 , such that the quotient $0_{K} / \mathcal{O}$ is finite, such an order must be of the form $\mathbb{O}=\mathbb{Z}\left[f \omega^{\prime}\right]$. The number $f$ is called the conductor of 0 . The discriminant of an order $0 \subset \mathbb{O}_{K}$ is equal to $D=f^{2} D_{k}$. Thus, the discriminant of an order is always congruent to 0 or 1 modulo 4 . The discriminant of the maximal order is called a fundamental discriminant.

For any discriminant, $D \equiv t(\bmod 4)$, the element $\omega=(t+\sqrt{D}) / 2$ is an algebraic integer since $t \equiv 0,1(\bmod 4)$. If we know that $D$ is fundamental then we usually write $\omega^{\prime}$ instead of $\omega$. With this notation, the expansion of $\omega_{n}=\left(1+\sqrt{S_{n}}\right) / 2$ corresponding to Shanks' sequence has a period length of $2 n+1$,

$$
\omega_{n}=\left[2^{n-1}+1, \overline{1,2^{n-1}, 2,2^{n-2}, 2^{2}, \ldots, 2^{n-1}, 1,2^{n}+1}\right] .
$$

The fundamental unit of the order with discriminant $D_{n}$ is given by

$$
\varepsilon_{n}=\left(\left(2^{n}+1+\sqrt{D_{n}}\right) / 2\right)\left(\left(2^{n}+3+\sqrt{D_{n}}\right) / 4\right)^{n} .
$$

The regulator of the order 0 , denoted by $R(O)$ or by $R(D)$ if 0 has discriminant $D$, is defined as the logarithm of the fundamental unit. Thus, sequences of discriminants $D_{n}$, where $\omega_{n}$ has a bounded period length have regulators of order $O\left(\log D_{n}\right)$. Examples like Shanks' sequence have regulators of order $O\left(\left(\log D_{n}\right)^{2}\right)$.

Several people have since generalized Shanks' sequence. They include Hendy [1974], Bernstein [1976a; 1976b; 1976c], Azuhata [1984; 1987], and Levesque and Rhin [1986]. A more synthetic account was given in [Williams 1985]. The most general form was presented in [Williams 1995] as

$$
D_{n}=\left(q r x^{n}+\mu\left(x^{k}-\lambda\right) / q\right)^{2}+4 \lambda r x^{n},
$$

with $\mu, \lambda \in\{-1,1\}$ and $r q \mid x^{k}-\lambda$. The automata of Raney were used in [van der Poorten 1994] to provide an alternate way of constructing $\omega_{n}$ and $\varepsilon_{n}$. 
Kaplansky [1998] coined the terms "sleepers", a sequence of discriminants whose period lengths are bounded, "creepers", a sequence whose lengths gently ${ }^{1}$ go to infinity, and "leapers", the generic discriminants whose period lengths increase exponentially.

By selecting a sequence of discriminants from families of sleepers appropriately, one can form a sequence of discriminants with linear period length. These are known as "beepers" and can be found in [Mollin and Cheng 2002; van der Poorten 1999; Williams and Buck 1994]. Since these discriminants are selected from sleepers they have a regulator of order $O\left(\log D_{n}\right)$.

These two ideas were used simultaneously by Madden [2001], who explicitly constructed a sequence of discriminants whose continued fraction expansions possessed slowly growing period lengths. These examples were distinct from the known creepers since they were not polynomially parametrized. However, they can be viewed as selecting specific discriminants from various families we will construct here, much as beepers are specially selected sleepers.

We define a creeper to be an infinite family of discriminants $D_{n}$, such that $f(X, n) \in \mathbb{Q}\left[X, X^{n}\right]$ and for a fixed $x \in \mathbb{Z}$ we have $D_{n}=f(x, n)$ satisfying

$$
l p\left(\omega_{n}\right)=a n+b \quad \text { with } a, b \in \mathbb{Q} \quad \text { and } \quad R\left(D_{n}\right)=\Theta\left(n^{2}\right) .
$$

Kaplansky [1998] made several conjectures about creepers which are quadratic in $x^{n}$. He suggested that every such creeper could be written as $D_{n}=A^{2} x^{2 n}+$ $B x^{n}+C^{2}$ with $A, B, C \in \mathbb{Q}$. Each of the examples upon which these conjectures were based has a principal ideal whose norm is a fixed power of $x$. Consequently, we define a kreeper to be an infinite sequence of discriminants $D_{n}$ such that

(1) $D_{n}=A^{2} x^{2 n}+B x^{n}+C^{2}$, where $A, B, C \in \mathbb{Q}$, and $x \in \mathbb{Z}^{+}$.

(2) $\operatorname{lp}\left(\omega_{n}\right)=a n+b$, where $a$ and $b$ are rational numbers.

(3) In the principal cycle there exists an element whose norm is $x^{g}$ for some $g$ fixed independently of $n$.

Note that the existence of some $Q_{h}=x^{g}$ implies $R\left(D_{n}\right)=\Theta\left(n^{2}\right)$. In other words, every kreeper is also a creeper. A proof of this is given in [Patterson 2003, Theorem 17]. Indeed many details are excluded here, and can be found in the same reference.

The main results here are the following.

Theorem 1.1. Any kreeper $D_{n}$ can be written as

$$
d^{2} D_{n}=c^{2}\left(\left(q r x^{n}+\left(m z^{2} x^{k}-l y^{2}\right) / q\right)^{2}+4 l y^{2} r x^{n}\right),
$$

\footnotetext{
${ }^{1}$ By "gently" he meant that the periods could be written in an arithmetic progression involving a parameter $n$ used in the presentation of the family.
} 
where each term in the above equation is an element of $\mathbb{Z}$, the terms $r, l, m$ are squarefree, $r, x$ are positive, and the following conditions hold:

$$
\begin{gathered}
(q r x, m l z y)=1, \quad(q r, x)=1, \quad(m z, l y)=1, \\
q\left|m z^{2} x^{k}-l y^{2}, \quad c^{2} r l y^{2} m z^{2}\right| d^{2} D_{n} .
\end{gathered}
$$

Theorem 1.2. Any sequence of discriminants given by (1-2) and satisfying the conditions (1-3) as above must in fact be a kreeper.

As a final introductory remark, we mention that Shanks' sequence has also been generalised to certain cubic fields with unit rank one, see [Adam 1995; 1998; Levesque and Rhin 1991].

\section{Preliminary results}

Removing nonpositive partial quotients from a continued fraction is not difficult, however removing a fractional partial quotient is, in general, quite difficult. The following few results do provide some assistance. Multiplication can be accomplished via

$$
x[a, b, c, d, \ldots]=[a x, b / x, x c, d / x, \ldots],
$$

which leads to:

Lemma 2.1 (Folding Lemma [Mendès France 1973]). Let $x / y=\left[a_{0}, a_{1}, \ldots, a_{h}\right]$ with $(x, y)=1$, and denote the sequence $a_{1}, \ldots, a_{h}$ by $\vec{w}$ (where $\overleftarrow{w}$ corresponds to the sequence $\left.a_{h}, \ldots, a_{1}\right)$. Then

$$
\frac{x}{y}+\frac{(-1)^{h}}{c y^{2}}=\left[a_{0}, \vec{w}, c-y^{\prime} / y\right]=\left[a_{0}, \vec{w}, c,-\overleftarrow{w}\right]
$$

where $y / y^{\prime}=\left[a_{h}, \ldots, a_{1}\right],\left(y, y^{\prime}\right)=1, y^{\prime}>0$.

This result is more than just a novelty. Besides our use of it here, in [van der Poorten 2002] it is used to rediscover the symmetry formulas.

A result which will be pivotal to our expansions later on is the following simple lemma.

Lemma 2.2. If $x / y=\left[a_{0}, \vec{w}\right]$, where $\vec{w}$ is defined as above then

$$
x / y+\gamma=\left[a_{0}, \vec{w}, \frac{(-1)^{h}}{\gamma y^{2}}-\frac{b}{y}\right],
$$

where $b$ is equal to $(-1)^{h+1} / x$ modulo $y$.

Proof. Using the Folding Lemma we obtain,

$$
\frac{x}{y}+\gamma=\frac{x}{y}+\frac{(-1)^{h}}{y^{2}}(-1)^{h} \gamma y^{2}=\left[a_{0}, \vec{w}, \frac{(-1)^{h}}{\gamma y^{2}}-\frac{b}{y}\right],
$$


where $b$ satisfies $x b-c y=(-1)^{h+1}$, which implies $b \equiv(-1)^{h+1} / x(\bmod y)$.

If the minimal polynomial of $\alpha$ is $x^{2}-t x+n$ then a typical line in the continued fraction expansion of $\alpha$ appears as

$$
\frac{\alpha+P_{h}}{Q_{h}}=a_{h}-\frac{\left(\bar{\alpha}+P_{h+1}\right)}{Q_{h}},
$$

where $P_{0}=0, Q_{0}=1$ and $\bar{\alpha}$ represents the nontrivial automorphism of the quadratic number field ${ }^{2}$. We then have

$$
P_{h+1}=a_{h} Q_{h}-P_{h}-t \quad \text { and } \quad Q_{h+1}=-\frac{n+P_{h}\left(P_{h}+t\right)}{Q_{h}}
$$

A quadratic irrational $\alpha$ is called reduced if $\alpha>1$ and $-1<\bar{\alpha}<0$. An integral ideal $\mathfrak{a}$ is called primitive if it is not divisible by any element of $\mathbb{Z}$. An integral ideal $\mathfrak{a}$ is reduced if there does not exist any nonzero $\alpha \in \mathfrak{a}$ satisfying

$$
|\alpha|<N(\mathfrak{a}) \quad \text { and } \quad|\bar{\alpha}|<N(\mathfrak{a}) .
$$

If $\mathfrak{a}$ is a primitive ideal such that $N(\mathfrak{a})<\sqrt{D} / 2$ then $\mathfrak{a}$ is reduced. From now on, ideal will mean "integral ideal".

One of the uses of the continued fraction expansion of a quadratic irrational is the determination of the fundamental unit. Rather than keeping track of the convergents, this can be done via the following result.

Proposition 2.3. If $\alpha=\left[a_{0}, a_{1}, \ldots, a_{i}, \alpha_{i+1}\right]$ and $x_{j} / y_{j}=\left[a_{0}, \ldots, a_{j}\right]$, where $\left(x_{j}, y_{j}\right)=1$, then

$$
\alpha_{1} \alpha_{2} \ldots \alpha_{h+1}=(-1)^{h+1}\left(x_{h}-y_{h} \alpha\right)^{-1} .
$$

Corollary 2.4. If $\mathbb{O}$ is an order of $\mathbb{Q}(\sqrt{D})$ and $\alpha_{i}, 1 \leq i \leq h+1$ represents a system of reduced elements in any cycle of quadratic irrationals in $\mathcal{O}$, then $\varepsilon=\prod_{i=1}^{h+1} \alpha_{i}$ is the fundamental unit of $\mathcal{O}$.

Such a cycle of quadratic irrationals is produced by the continued fraction expansion. To be more precise, if $\mathfrak{a}_{0}=Q_{0} \mathbb{Z}+\left(P_{0}+\omega\right) \mathbb{Z}$ is an ideal of 0 then the continued fraction expansion of $\left(\omega+P_{0}\right) / Q_{0}$ produces a sequence of complete quotients $\left(\omega+P_{i}\right) / Q_{i}$ such that the ideals associated to each complete quotient, that is $\mathfrak{a}_{i}=Q_{i} \mathbb{Z}+\left(\omega+P_{i}\right) \mathbb{Z}$, are all equivalent to $\mathfrak{a}_{0}$.

Later we will need to transfer results from one order to another, where the following proposition will be useful.

\footnotetext{
${ }^{2}$ The $P_{h}, Q_{h}$ appearing here are not, in general, the same as those used in [Perron 1950]
} 
Proposition 2.5. Let $\mathrm{O}_{1}, \mathrm{O}_{2}$ be two orders of a real quadratic field given by $\mathrm{O}_{1}=$ $\mathbb{Z}\left[f \omega^{\prime}\right], \mathrm{O}_{2}=\mathbb{Z}\left[g \omega^{\prime}\right]$ then

$$
R\left(\mathrm{O}_{1}\right)>\frac{(g, f)}{2 g} R\left(\mathrm{O}_{2}\right) .
$$

Let $\mathfrak{a}$ be any ideal of the order $\mathcal{O}$ having conductor $f$ in $K$, and suppose that $(N(\mathfrak{a}), f)=1$. Then $\mathfrak{a}=(t) \mathfrak{r} \mathfrak{s}$, where $t \in \mathbb{Z}$ and any prime ideal divisor of $\mathfrak{r}$ lies over a prime which ramifies and any prime ideal divisor of $\mathfrak{s}$ lies over a prime which splits in $\mathcal{O}$. Furthermore, $\mathfrak{r}$ and $\mathfrak{s}$ are primitive. We will denote by $s(\mathfrak{a})$ the ideal $\mathfrak{s}$. Note that if $t=1$ (in which case $\mathfrak{a}$ is primitive) then $\mathfrak{a}^{2}=(r) \mathfrak{s}^{2}$, where $r$ is squarefree and $r \mid D$. Also note that $N(s(\mathfrak{a}))=N(s(\overline{\mathfrak{a}}))$.

We now introduce a generalisation of a result of Yamamoto [1971].

Definition 1. Let $\mathfrak{b}_{1}, \ldots, \mathfrak{b}_{n}$ be invertible ideals of an order $\mathfrak{O}$. We say that $\mathfrak{b}_{1}, \ldots, \mathfrak{b}_{n}$ are independent in 0 if whenever there exist nonzero integers $u, v$ and $\alpha_{i}, \beta_{i} \in \mathbb{Z}^{+}(i=1, \ldots, n)$ such that

$$
\text { (u) } \prod_{i=1}^{n} \mathfrak{b}_{i}^{\alpha_{i}}=(v) \prod_{i=1}^{n} \mathfrak{b}_{i}^{\beta_{i}}
$$

then $\alpha_{i}=\beta_{i}(i=1, \ldots, n)$.

A sufficient condition for the independence of two ideals $\mathfrak{b}$ and $\mathfrak{c}$ in $\mathbb{O}$ is given in Theorem 2.7, which needs the following Lemma.

Lemma 2.6. If $\mathfrak{b}$ and $\mathfrak{c}$ are dependent in 0 then there exist nonzero integers $u, v$ and nonnegative integers $m, n$, with $m+n>0$, such that

$$
(u) \mathfrak{b}^{m}=(v) \mathfrak{d}^{n},
$$

where $\mathfrak{d}$ is equal to $\mathfrak{c}$ or $\overline{\mathfrak{c}}$.

Theorem 2.7. If $\mathfrak{b}$ and $\mathfrak{c}$ are dependent in $\mathcal{O}$ and $(N(\mathfrak{b}) N(\mathfrak{c}), f)=1$ then for some nonnegative integers $m, n$, with $m+n>0$ we have

$$
N(s(\mathfrak{b}))^{m}=N(s(\mathfrak{c}))^{n} .
$$

Proof. By the preceding Lemma, we know there must exist integers $m, n, u, v$ such that $(u) \mathfrak{b}^{m}=(v) \mathfrak{d}^{n}$, where $m, n$ are nonnegative and at least one of $m, n$ is positive. Then we can write $\left(u t_{1}^{m}\right) \tilde{\mathfrak{b}}^{m}=\left(v t_{2}^{n}\right) \tilde{\mathfrak{d}}^{n}$, where $\tilde{\mathfrak{b}}$ and $\tilde{\mathfrak{d}}$ are primitive.

The condition $(f, N(\mathfrak{b}) N(\mathfrak{c}))=1$ and primitivity allow us to write

$$
\left(u t_{1}^{m}\right) \tilde{\mathfrak{b}}^{m}=\left(u t_{1}^{m}\right) r(\mathfrak{b})^{m} s(\mathfrak{b})^{m} \quad \text { and } \quad\left(v t_{2}^{n}\right) \tilde{\mathfrak{d}}^{n}=\left(v t_{2}^{n}\right) r(\mathfrak{d})^{n} s(\mathfrak{d})^{n} .
$$

Squaring these yields

$$
\left(u^{2} r_{1}^{m} t_{1}^{2 m}\right) s(\mathfrak{b})^{2 m}=\left(v^{2} r_{2}^{n} t_{2}^{2 n}\right) s(\mathfrak{d})^{2 n},
$$


where $r_{1}$ and $r_{2}$ divide $D_{n}$. Dividing out any common factors of $u^{2} r_{1}^{m} t_{1}^{2 m}$ and $v^{2} r_{2}^{n} t_{2}^{2 n}$ provides coprime integers $u_{1}, v_{1}$ such that

$$
\left(u_{1}\right)(s(\mathfrak{b}))^{2 m}=\left(v_{1}\right)(s(\mathfrak{d}))^{2 n} .
$$

Let $\mathfrak{p}^{\alpha} \| s(\mathfrak{b})$ and $\mathfrak{p}^{\beta} \| s(\mathfrak{d})$. If $\mathfrak{p}^{2 m \alpha} \not\langle s(\mathfrak{d})$ then $N(\mathfrak{p})| v_{1}$ implies that $\overline{\mathfrak{p}} \mid\left(v_{1}\right)$. The coprimality of $u_{1}$ and $v_{1}$ means $\overline{\mathfrak{p}} \mid(s(\mathfrak{b}))^{2 m}$, which gives $N(\mathfrak{p}) \mid s(\mathfrak{b})$, which is impossible because $s(\mathfrak{b})$ is primitive.

Hence, $\mathfrak{p}^{2 m \alpha} \mid s(\mathfrak{d})$, which means that $2 m \alpha \leq 2 n \beta$. By symmetry, $2 n \beta \leq 2 m \alpha$ and so $m \alpha=n \beta$. Thus, $s(\mathfrak{b})^{m}=s(\mathfrak{d})^{n}$ and we find $N(s(\mathfrak{b}))^{m}=N(s(\mathfrak{d}))^{n}$.

Suppose that $\mathfrak{b}_{1}, \ldots, \mathfrak{b}_{n}$ are independent ideals of $\mathscr{O}$ of discriminant $D$ and let

$$
S=\left\{\prod_{i=1}^{n} \mathfrak{b}_{i}^{\alpha_{i}}: \alpha_{i} \geq 0 i=1, \ldots, n \text { and } \prod_{i=1}^{n} N\left(\mathfrak{b}_{i}\right)^{\alpha_{i}}<\sqrt{D} / 2\right\}
$$

If $\mathfrak{a}_{i}=Q_{i-1} \mathbb{Z}+\left(\omega+P_{i-1}\right) \mathbb{Z}$ is a reduced ideal then

$$
\frac{\omega+P_{i-1}}{Q_{i-1}}>\frac{\sqrt{D} / 2}{Q_{i-1}}=\frac{\sqrt{D} / 2}{\left|N\left(\mathfrak{a}_{i}\right)\right|} .
$$

Now suppose $(v) \mathfrak{a} \in S$, where $\mathfrak{a}$ is reduced and $\mathfrak{a}=\left\langle Q_{i-1}, \omega+P_{i-1}\right\rangle$. We have

$$
\frac{\omega+P_{i-1}}{Q_{i-1}}>\frac{v^{2} \sqrt{D} / 2}{\prod_{i=1}^{n} N\left(\mathfrak{b}_{i}\right)^{\alpha_{i}}} \geq \frac{\sqrt{D} / 2}{\prod_{i=1}^{n} N\left(\mathfrak{b}_{i}\right)^{\alpha_{i}}} .
$$

Theorem 2.8. Let $\mathrm{O}_{1}, \mathrm{O}_{2}, \ldots$, be a sequence of orders, each of discriminant $D_{i}$, where $D_{i}<D_{i+1}$. Suppose further that in each $\mathrm{O}_{i}$ there exists an independent set of principal ideals $\left\{\mathfrak{b}_{i, j}:(j=1, \ldots, n)\right\}$ such that $N\left(\mathfrak{b}_{i, j}\right)$ is fixed for each value of $i$. Then

$$
R\left(D_{i}\right) \gg\left(\log D_{i}\right)^{n+1}
$$

See [Patterson 2003] for a proof.

\section{Basic observations on kreepers}

Given discriminants of the form $D_{n}=U^{2} x^{2 n}+V x^{n}+W^{2}$, where $U, V, W \in \mathbb{Q}$, there is no loss in generality in supposing that $x$ is not a power. We may write our discriminants $D_{n}$ as

$$
D_{n}=\frac{c^{2}}{d^{2}}\left[\left(A x^{n}+C\right)^{2}+4 G x^{n}\right], \quad \text { where } G=(B-2 A C) / 4,
$$

for $A, B, C, G, c, d \in \mathbb{Z}$ and $(c, d)=1$. 
Any common factors of $C$ and $x$ can be moved into the square divisors. By considering

$$
W:=\max _{i \in \mathbb{N}}\left(x^{i}, C\right), \quad m:=\min \left\{i \in \mathbb{N}:\left(x^{i}, C\right)=W\right\}, \quad v:=n-2 m,
$$

so that $(x, C / W)=1$, we have

$$
D_{n}=\frac{c^{2} W^{2}}{d^{2}}\left(\left(A W \frac{x^{2 m}}{W^{2}} x^{v}+\frac{C}{W}\right)^{2}+4 G \frac{x^{2 m}}{W^{2}} x^{v}\right)=\frac{c^{2} W^{2}}{d^{2}}\left(\left(\bar{A} x^{v}+\bar{C}\right)^{2}+4 \bar{G} x^{v}\right),
$$

where $(x, \bar{C})=1$ and $\bar{A}, \bar{C}, \bar{G} \in \mathbb{Z}$. Any square factors of $\left(A^{2}, C^{2}, G\right)$ can also be removed, so that without loss of generality we may suppose that

$$
D_{n}=\left(\frac{c}{d}\right)^{2}\left(\left(A x^{n}+C\right)^{2}+4 G x^{n}\right)
$$

with $A, C, G \in \mathbb{Z},(x, C)=1$ and $\left(A^{2}, C^{2}, G\right)$ is squarefree.

The next few results don't use any of the properties of kreepers, we are merely interested in determining an explicit formula for $\left(A x^{n}+C\right)^{2}+4 G x^{n}$. Consequently, we define

$$
E_{n}:=\left(A x^{n}+C\right)^{2}+4 G x^{n},
$$

where $(x, C)=1$ and $\left(A^{2}, C^{2}, G\right)$ is squarefree. The first result is a representation of $A, C, G$.

Theorem 3.1. Given $E_{n}$ as in (3-2) and the conditions on A, C, G, x stated above, we have that

$$
E_{n}=\left(q r N x^{n}+P(M-L) / q\right)^{2}+4 r L N P x^{n},
$$

where $r, q, P, N \in \mathbb{Z}^{+}, M, L \in \mathbb{Z}$, and the following conditions are satisfied:
(1) $r$ is squarefree,
(2) $(P, r q N)=1$,
(3) $r q \mid M-L$,
(4) $(M, L)=1$,
(5) $(r q, M L)=1$,
(6) $(r, L N P)=1$.

Proof. The selections we make are

$$
\begin{aligned}
r:=(A, C, G), & N:=\left(\frac{A}{r}, \frac{G}{r}\right), & q:=\frac{A}{r N}, \\
P:=\left(\frac{C}{r}, \frac{G}{N r}\right), & L:=\frac{G}{r N P}, & M:=\frac{A C+G}{r N P} .
\end{aligned}
$$

These selections make (3-2) and (3-3) equivalent, so it only remains to show that the conditions indicated hold. This is not difficult; see [Patterson 2003].

Our next objective is to determine the terms that divide $E_{n}$ and those that are coprime to $E_{n}$. 
Lemma 3.2. $\left(E_{n}, x N P\right)=1$.

Proof. See [Patterson 2003].

Theorem 3.3. For the family of discriminants given by (3-3) with $n \in I$ (an infinite subset of $\mathbb{N}$ ) there exists an infinite set $I^{\prime} \subseteq I$ such that for every $n \in I^{\prime}$ we have $M=v^{\prime} m z^{2} Z, L=v l y^{2} Y$ with $Z, Y, z, y$ positive and

$$
m l\left|E_{n}^{\prime}, \quad v v^{\prime} z y\right| F_{n}, \quad v v^{\prime} \mid 2, \quad\left(Z Y, E_{n}^{\prime}\right)=1, \quad\left(\frac{F_{n}}{z y}, m l Z Y\right)=1
$$

and $E_{n}=F_{n}^{2} E_{n}^{\prime}$, where $E_{n}^{\prime}$ is squarefree. Here $m, l, z, y, Z, Y$ are the same for all $n \in I^{\prime}$.

Proof. Define $y_{n}^{2} v_{n}:=\left(F_{n}^{2}, L\right)$, where $v_{n}$ is squarefree. Next, write

$$
\tilde{F}_{n}=\frac{F_{n}}{y_{n} v_{n}} \quad \text { so that }\left(v_{n} \tilde{F}_{n}^{2}, \frac{L}{v_{n} y_{n}^{2}}\right)=1
$$

and

$$
E_{n}=y_{n}^{2} v_{n}^{2} \tilde{F}_{n}^{2} E_{n}^{\prime}=S_{n}^{2}+4 r v_{n} y_{n}^{2} \frac{L}{v_{n} y_{n}^{2}} N P x^{n}
$$

where

$$
S_{n}=q r N x^{n}+\frac{P(M-L)}{q} .
$$

It is not difficult to show that $v_{n} \mid 2$; see [Patterson 2003].

We now investigate the factors of $L / v_{n} y_{n}^{2}$. (Since $\left(F_{n} /\left(v_{n} y_{n}\right), L /\left(v_{n} y_{n}^{2}\right)\right)=1$ it follows that for any prime factor, $p$, of $L / v_{n} y_{n}^{2}$, we have $p \mid S_{n} /\left(v_{n} y_{n}\right)$ if and only if $p \mid E_{n}^{\prime}$ ). We define $l_{n}$ to be the product of all prime powers $p^{\alpha}$ of $L / v_{n} y_{n}^{2}$ that satisfy $p^{\alpha} \| L / v_{n} y_{n}^{2}$ and $p \mid E_{n}^{\prime}$, and $Y_{n}$ to be the product of all prime powers $p^{\beta}$ that satisfy $p^{\beta} \| L / v_{n} y_{n}^{2}$ and $\left(E_{n}^{\prime}, p\right)=1$. We also absorb the sign of $L$ into $l_{n}$. Clearly, $L / v_{n} y_{n}^{2}=l_{n} Y_{n}$. It is straightforward to show that $l_{n}$ is squarefree and so we find $L=v_{n} l_{n} y_{n}^{2} Y_{n},\left(Y_{n}, E_{n}^{\prime}\right)=1, v_{n} y_{n}\left|F_{n}, l_{n}\right| E_{n}^{\prime}, v_{n} \mid 2$.

Equation (3-4) can also be written as

$$
E_{n}=z_{n}^{2}\left(v_{n}^{\prime}\right)^{2} \tilde{F}_{n}^{2} E_{n}^{\prime}=\left(S_{n}^{\prime}\right)^{2}+4 r v_{n}^{\prime} z_{n}^{2} \frac{M}{v_{n}^{\prime} z_{n}^{2}} N P x^{n},
$$

where $v_{n}^{\prime}$ is squarefree and

$$
S_{n}^{\prime}=q r N x^{n}-P(M-L) / q \quad \text { and } \quad z_{n}^{2} v_{n}^{\prime}=\left(F_{n}^{2}, M\right) .
$$

By similar reasoning we get $M=m_{n} v_{n}^{\prime} z_{n}^{2} Z_{n},\left(Z_{n}, E_{n}^{\prime}\right)=1, v_{n}^{\prime} z_{n}\left|F_{n}, m_{n}\right| E_{n}^{\prime}$, $v_{n}^{\prime} \mid 2$, and $4 \nmid v_{n} v_{n}^{\prime}$ because $(M, L)=1$. 
As there are only a finite number of choices for $m_{n}, l_{n}, z_{n}, y_{n}, v_{n}, v_{n}^{\prime}, Z_{n}, Y_{n}$ for fixed values of $L$ and $M$ there must exist some infinite set $I^{\prime} \subseteq I$ for which

$$
M=v^{\prime} m z^{2} Z, \quad L=v l y^{2} Y, \quad m l\left|E_{n}^{\prime}, \quad z y v v^{\prime}\right| F_{n}, \quad\left(Z Y, E_{n}^{\prime}\right)=1, \quad v v^{\prime} \mid 2 .
$$

The coprime conditions all follow easily.

This completes our investigation of $E_{n}$; we have determined the factors coprime to $E_{n}$ and those which divide each part of it.

\section{Independent ideals in kreepers}

Completing the square on $A^{2} E_{n}$ gives,

$$
A^{2} E_{n}=\left(A^{2} x^{n}+B / 2\right)^{2}-4 G H
$$

and $B / 2$ is an integer because $4 \mid(B-2 A C)$. In terms of the constants found in the previous section, this equation becomes

$$
q^{2} F_{n}^{2} E_{n}^{\prime}=\left(q^{2} r N x^{n}+P(L+M)\right)^{2}-4 P^{2} L M
$$

We define $\omega_{n}^{\prime}:=\left(\sigma_{n}^{\prime}-1+\sqrt{E_{n}^{\prime}}\right) / \sigma_{n}^{\prime}$, where $E_{n}^{\prime}$ is squarefree and $\sigma_{n}^{\prime}=2$ if $E_{n}^{\prime} \equiv 1$ $(\bmod 4)$ and $\sigma_{n}^{\prime}=1$ otherwise. We further define $\sigma_{n}=\mathbb{Z}+\omega_{n} \mathbb{Z}$, where

$$
\omega_{n}:=\frac{t_{n}+\sqrt{D_{n}}}{2} \quad \text { and } \quad t_{n}=\left\{\begin{array}{lll}
0 & \text { if } D_{n} \equiv 0 & (\bmod 4) \\
1 & \text { if } D_{n} \equiv 1 & (\bmod 4)
\end{array}\right.
$$

Proposition 4.1. $O_{n}=\mathbb{Z}+\frac{c F_{n} \sigma_{n}^{\prime}}{2 d} \omega_{n}^{\prime} \mathbb{Z}$.

Our objective now is to find an ideal arising from (4-1) which has a norm coprime to the conductor of some order.

Proposition 4.2. For each $n$, there exists an element $\alpha_{n} \in \mathbb{Z}+f_{1} \omega_{n}^{\prime} \mathbb{Z}$, where $f_{1}=F_{n} /(\lambda z y)$, and $\lambda=1$ or 2 , and $\left(N\left(\alpha_{n}\right), f_{1}\right)=1$.

Proof. We take $\alpha_{n}$ as

$$
\alpha_{n}:= \begin{cases}s_{n} / 2-\frac{q F_{n}}{2 z y}+\frac{q F_{n}}{z y} \omega_{n}^{\prime} & \text { if } 2 \nmid q \text { and } 2 \nmid F_{n} / z y, \\ s_{n} / 2+\frac{q F_{n}}{2 z y} \sqrt{E_{n}^{\prime}} & \text { otherwise. }\end{cases}
$$

The remaining details are in [Patterson 2003]. 
Bounded norms in kreepers. By the definition of a kreeper, the continued fraction expansion of $\omega_{n}$ has some $Q_{i}=x^{g}$ with $g$ fixed independently of $n$. In other words, there exists some $\mu_{n} \in \mathcal{O}_{n}$ such that $N\left(\mu_{n}\right)=x^{g}$. Also recall Proposition 4.1, which states that $\mathcal{O}_{n}=\mathbb{Z}+f_{2} \omega_{n}^{\prime} \mathbb{Z}$, where $f_{2}=c F_{n} \sigma_{n}^{\prime} / 2 d$. Let $f_{3}=\left(f_{1}, f_{2}\right)$, so that $\alpha_{n}$, $\mu_{n}$ are both contained in $O_{n}^{*}:=\mathbb{Z}+f_{3} \omega_{n}^{\prime} \mathbb{Z}$. We know that $\left(x, E_{n}\right)=1$, so then $\left(x, F_{n} / z y\right)=1$. Hence,

$$
\left(N\left(\alpha_{n}\right), f_{3}\right)=\left(N\left(\mu_{n}\right), f_{3}\right)=1 .
$$

Thus in the order $\mathbb{O}_{n}^{*}$ we have the two principal ideals $\mathfrak{a}_{n}=\left(\alpha_{n}\right)$ and $\mathfrak{b}_{n}=\left(\mu_{n}\right)$ (whose norms are fixed for infinitely many $n \in I$ ). Both ideals have norms coprime to the conductor. Hence, we may apply Theorems 2.7 and 2.8.

Proposition 4.3. If $\mathfrak{a}_{n}$ and $\mathfrak{b}_{n}$ are independent ideals in $\mathfrak{O}_{n}^{*}$ then

$$
R\left(\mathscr{O}_{n}\right) \gg\left(\log D_{n}\right)^{3} .
$$

Proof. By Theorem 2.8,

$$
R\left(\mathbb{O}_{n}^{*}\right) \gg\left(\log \Delta\left(\mathbb{O}_{n}^{*}\right)\right)^{3}=\left(\log \left(f_{3}^{2} \Delta\left(\mathbb{O}_{n}^{\prime}\right)\right)\right)^{3},
$$

where $\mathbb{O}_{n}^{\prime}:=\mathbb{Z}+\omega_{n}^{\prime} \mathbb{Z}$ and $\Delta(\mathcal{O})$ denotes the discriminant of the order $\mathbb{O}$. By Proposition 2.5,

(4-2) $R\left(\mathscr{O}_{n}\right)>\frac{\left(f_{3}, f_{2}\right)}{2 f_{3}} R\left(\mathscr{O}_{n}^{*}\right)$ implies $R\left(\mathscr{O}_{n}\right)>\frac{1}{2} R\left(\mathscr{O}_{n}^{*}\right) \gg\left(\log \left(f_{3}^{2} \Delta\left(\mathscr{O}_{n}^{\prime}\right)\right)\right)^{3}$.

Since $f_{3}=F_{n}\left(2 d, c \sigma_{n}^{\prime} z y \lambda\right) / 2 \lambda z y d$ we find

$$
\left(\log f_{3}^{2} \Delta\left(\mathbb{O}_{n}^{\prime}\right)\right)^{3} \gg\left(\log F_{n}^{2} \Delta\left(\mathscr{O}_{n}^{\prime}\right)\right)^{3} \gg\left(\log F_{n}^{2} E_{n}^{\prime}\right)^{3} \gg\left(\log D_{n}\right)^{3} .
$$

Hence, from (4-2), we have that $R\left(\mathrm{O}_{n}\right) \gg\left(\log D_{n}\right)^{3}$.

Consequently, in order for the sequence of discriminants $\left\{D_{n}\right\}$ to be a kreeper, $\mathfrak{a}_{n}$ and $\mathfrak{b}_{n}$ must be dependent ideals in $\mathbb{O}_{n}^{*}$. By Theorem 2.7 there must exist nonnegative integers $e$ and $f$, with $e$ and $f$ not both 0 such that $N\left(s\left(\mathfrak{a}_{n}\right)\right)^{e}=N\left(s\left(\mathfrak{b}_{n}\right)\right)^{f}$.

With not too much effort (see [Patterson 2003, Chapter V, §15]) one shows that $N\left(s\left(\mathfrak{a}_{n}\right)\right)=P^{2} Z Y$ and $N\left(s\left(\mathfrak{b}_{n}\right)\right)=x^{g}$. If $e=0$ then $x^{g f}=1$ and since in this case we must have $f>0$ we find $x=1$, which is impossible. On the other hand, if $f=0$ then $Y, Z$ and $P$ are all \pm 1 , in which case

$$
\frac{q^{2} E_{n}}{(z y)^{2}}=s_{n}^{2} \pm 4 m l v v^{\prime}
$$

But $m l v v^{\prime}$ divides $E_{n} /(z y)^{2}$, which by a result of Schinzel [1961] implies that the period length of $q^{2} E_{n} /(z y)^{2}$ is bounded for all $n$. In the terminology of [Kaplansky 1998], this says that $q^{2} E_{n} /(z y)^{2}$ is a sleeper. It is shown in [Patterson 
2003, Chapter III] that any rational multiple of a sleeper is again a sleeper. Hence, $c^{2} E_{n} / d^{2}=D_{n}$ must also be a sleeper; in other words, $D_{n}$ is not a kreeper. It follows that if $D_{n}$ is to be a kreeper we must have $N\left(s\left(\mathfrak{a}_{n}\right)\right)^{e}=N\left(s\left(\mathfrak{b}_{n}\right)\right)^{f}$ with $e, f$ each being positive. Since $(x, P)=1$ we must have $P= \pm 1$. Hence, we may replace $P M$ by $M$ and $P L$ by $L$ and all the previous conditions hold. Taking $d:=(g f, e), k:=g f / d, h:=e / d$ gives,

$$
(Z Y)^{h}=x^{k}, \quad(h, k)=1,
$$

which implies that $x=R^{h}, Z Y=R^{k}$. Hence, $h=1$ and $Z Y=x^{k}$ because $x$ is not a power. From Theorem 3.1 we have $(Z, Y)=1$; hence

$$
Z=U^{k}, \quad Y=T^{k}, \quad \text { where }(U, T)=1 .
$$

The objective of the next 2 sections will be to show that $T=1$ and $U=x$.

\section{Part of the continued fraction expansion of $\omega_{n}$}

There is no longer any need to distinguish between factors of $E_{n}^{\prime}$ and $F_{n}$, which means that we may absorb the terms $v$ and $v^{\prime}$ into $l$ and $m$ respectively. The form of a kreeper is now given by

$$
D_{n}=\left(\frac{c}{d}\right)^{2}\left(\left(q r N(U T)^{n}+\left(z^{2} m U^{k}-y^{2} l T^{k}\right) / q\right)^{2}+4 r N l y^{2} T^{k+n} U^{n}\right),
$$

where $m, l, r$ are squarefree and

$$
(q r, U T)=1, \quad(q r N U T, y z m l)=1, \quad(T y l, m z U)=1, q r \mid z^{2} m U^{k}-y^{2} l T^{k}
$$

and for every $n \in I$,

$$
y l \mid q^{2} r N x^{n}+z^{2} m U^{k} \text { and } m z \mid q^{2} r N x^{n}+y^{2} l T^{k},
$$

and $N>0, x=U T$. Let $\mu$ be the least positive difference of any two integers in $I$. Then $v, v+\mu \in I$ for some $v$, and

$$
y l \mid q^{2} r N x^{\nu}+z^{2} m U^{k} \quad \text { and } \quad y l \mid q^{2} r N x^{\nu+\mu}+z^{2} m U^{k},
$$

which means $y l \mid q^{2} r N x^{\nu}\left(x^{\mu}-1\right)$, so $y l \mid x^{\mu}-1$ because $(y l, q r N x)=1$. Вy symmetry, $z m \mid x^{\mu}-1$. Thus, $y \operatorname{lm} z \mid x^{\mu}-1$. Hence the conditions (5-2) become $y l\left|q^{2} r N(U T)^{\nu}+z^{2} m U^{k}, m z\right| q^{2} r N(U T)^{\nu}+y^{2} l T^{k},(T U)^{\mu} \equiv 1 \quad(\bmod y \operatorname{lm} z)$

for any $n \in I$, such that $n \equiv v(\bmod \mu)$. Since the signs of $m$ and $l$ have not yet been specified, there is no loss of generality in supposing that $q, U, T$ are all positive. 
Since (5-1) can also be represented as

$$
D_{n}=\left(\frac{c}{d}\right)^{2}\left(\left(q r N(U T)^{n}-\left(z^{2} m U^{k}-y^{2} l T^{k}\right) / q\right)^{2}+4 r N m z^{2} T^{n} U^{k+n}\right)
$$

we may assume without loss of generality that $U>T$.

Some notation. We now rewrite equations (5-1) and (5-3) as

$$
D_{n}=\left(\frac{s_{1}}{d}\right)^{2}+4\left(\frac{c}{d}\right)^{2} r N l y^{2} T^{k+n} U^{n}=\left(\frac{s_{2}}{d}\right)^{2}+4\left(\frac{c}{d}\right)^{2} r N m z^{2} T^{n} U^{k+n},
$$

where

$$
s_{1}=c q r N x^{n}+c\left(z^{2} m U^{k}-y^{2} l T^{k}\right) / q, \quad s_{2}=c q r N x^{n}-c\left(z^{2} m U^{k}-y^{2} l T^{k}\right) / q .
$$

We choose an infinite subset of $I$ such that $t \equiv D_{n}(\bmod 4)$ is fixed. Then we take $S_{1}:=\left(s_{1}-t d\right) / 2$ and $S_{2}:=\left(s_{2}-t d\right) / 2$. We also write

$$
\alpha=\omega_{n}+S_{1} / d, \quad \bar{\alpha}=\bar{\omega}_{n}+S_{1} / d \quad \text { and } \quad \beta=\omega_{n}+S_{2} / d, \quad \bar{\beta}=\bar{\omega}_{n}+S_{2} / d .
$$

Then

$$
\alpha \bar{\alpha}=-c^{2} r N l y^{2} T^{k} x^{n} / d^{2} \quad \text { and } \quad \beta \bar{\beta}=-c^{2} r N m z^{2} U^{k} x^{n} / d^{2} .
$$

Further, we have

$$
q^{2} d^{2} D_{n}=\left(q s_{3}\right)^{2}-4 c^{2} m l(z y)^{2}(U T)^{k},
$$

where

$$
q s_{3}=c q^{2} r N x^{n}+m z^{2} U^{k}+l y^{2} T^{k} .
$$

Also of relevance will be $S_{1}+S_{2}+t d=A x^{n}=c q r N x^{n}$.

We now detail an initial segment of the continued fraction expansion of $\omega_{n}$. In the case of $T>1$, this segment will have length $O\left(n^{1+\varepsilon}\right)$, hence the entire expansion could not satisfy $\operatorname{lp}\left(\omega_{n}\right)=a n+b$, as required by kreepers.

Before commencing we need to determine the common factors between some of the terms. First, we define $g:=\left(s_{1}, s_{2}, d\right)$. It is not to difficult to show that $\left(z, s_{1} / c\right)$ and $\left(y, s_{2} / c\right)$ each divides 2 . We also define $d_{y}:=\left(S_{1}, d\right), d_{y}^{\prime}:=\left(s_{1} / g, d / g\right)$ and $\tau_{y}:=d_{y} / d_{y}^{\prime}$. It follows easily that $\tau_{y}$ is an integer. Similarly, we define $d_{z}:=\left(S_{2}, d\right)$, $d_{z}^{\prime}:=\left(s_{2} / g, d / g\right)$ and $\tau_{z}:=d_{z} / d_{z}^{\prime}$. Next, we write $d=\bar{d} d_{z} d_{y}$. Here are some simple results:

- $g \mid 2$, moreover, $g=2$ if and only if $2 \mid d$.

- $\tau_{z} \mid g$ and $\tau_{y} \mid g$.

- $\tau_{y} d_{y}^{\prime} \mid y$ and $\tau_{z} d_{z}^{\prime} \mid z$; in other words $d_{y} \mid y$ and $d_{z} \mid z$. 
Expansion of $\omega_{n}$. The continued fraction expansion of $\omega_{n}$ begins as

$$
\omega_{n}=\frac{\left(S_{1}+t d\right) / d_{y}}{d / d_{y}}-\left(\bar{\omega}_{n}+S_{1} / d\right)
$$

and $\left(\left(S_{1}+t d\right) / d_{y}, d / d_{y}\right)=1$ by the definition of $d_{y}$. Hence we may apply Lemma 2.2 , and find that after the expansion of $\left(S_{1}+t d\right) / d$, of length $h_{0}$, a new complete quotient in the continued fraction expansion is

$$
\frac{-(-1)^{h_{0}+1}}{\left(\bar{\omega}_{n}+S_{1} / d\right)\left(d / d_{y}\right)^{2}}-\frac{c_{0}}{d / d_{y}}
$$

where

$$
c_{0} \equiv \frac{(-1)^{h_{0}}}{\left(S_{1}+t d\right) / d_{y}} \quad\left(\bmod d / d_{y}\right)
$$

By choosing $(-1)^{h_{0}+1}=\operatorname{sign}(l)$ the element in (5-4) then becomes

$$
\frac{\omega_{n}+S_{1} / d}{c^{2} r N|l| y^{2} T^{k} x^{n} /\left(d_{y}\right)^{2}}-\frac{c_{0}}{d / d_{y}}=\frac{\omega_{n}+S_{1} / d-c_{0} c^{2} r N|l| d_{y}\left(y / d_{y}\right)^{2} T^{k} x^{n} / d}{c^{2} r N|l|\left(y / d_{y}\right)^{2} T^{k} x^{n}} .
$$

Now define: $s:=\max _{i \in \mathbb{N}}\left\{\left(x^{i}, c\right)\right\}$ and $u:=c / s$. Hence $(u, x)=1$. Recall, $(x, q r z y m l)=1$ so then $(s, q r z y m l)=1$. We will denote the element in (5-5) as $\theta_{h_{0}}$. From it, we can write

$$
\theta_{h_{0}}=\frac{\omega_{n}+P_{h_{0}}}{Q_{h_{0}}}=\frac{A_{0}}{B_{0}}-\frac{\bar{\beta}}{c^{2} r N|l|\left(y / d_{y}\right)^{2} T^{k} x^{n}},
$$

where

$$
A_{0}=\operatorname{cqr} N x^{n}-c_{0} c^{2} r N|l| d_{y}\left(y / d_{y}\right)^{2} T^{k} x^{n}, \quad B_{0}=c^{2} d r N|l|\left(y / d_{y}\right)^{2} T^{k} x^{n} .
$$

Next, we define $\Delta_{0}:=\left(A_{0}, B_{0}\right)$. We need to determine $\Delta_{0}$ before we can apply Lemma 2.2 again. One finds,

$$
\Delta_{0}=\operatorname{crN} x^{n} \delta d_{z} \quad \text { and } \quad B_{0} / \Delta_{0}=c / \delta d / d_{z}|l|\left(y / d_{y}\right)^{2} T^{k} .
$$

From (5-6), by applying Lemma 2.2, we find the next partial quotients are those of the continued fraction expansion of $A_{0} / B_{0}$ of length $p_{0}$. By choosing $(-1)^{p_{0}+1}=$ $\operatorname{sign}(m)$, the next element in the continued fraction expansion is $\theta_{h_{1}}$, where $h_{1}:=$ $h_{0}+p_{0}$,

$$
\theta_{h_{1}}=\frac{c^{2} r N|l|\left(y / d_{y}\right)^{2} T^{k} x^{n}}{-\bar{\beta} \operatorname{sign}(m)\left(B_{0} / \Delta_{0}\right)^{2}}-\frac{c_{1}}{B_{0} / \Delta_{0}}
$$


and $c_{1} \equiv-\operatorname{sign}(m) \Delta_{0} / A_{0} \quad\left(\bmod B_{0} / \Delta_{0}\right)$. We can write

$$
\theta_{h_{1}}=\frac{\omega_{n}+S_{2} / d-c_{1}(c / \delta)|m| z\left(z / d_{z}\right) U^{k} / d}{(c / \delta)^{2}|m l|\left(y / d_{y}\right)^{2}\left(z / d_{z}\right)^{2}(U T)^{k}} .
$$

Finding other complete quotients. The set of conditions

$$
\begin{gathered}
u_{2 i-1}\left|u, \quad z_{2 i-1}\right| z / d_{z}, \quad z_{2 i-1}^{\prime} \mid z, \quad\left(c_{2 i-1}, s T\right)=1, \quad\left(z_{2 i-1} y_{2 i-1}, u / u_{2 i-1}\right)=1, \\
m_{2 i-1} \in\{1,|m|\}, \quad l_{2 i-1} \in\{1,|l|\}, \quad r_{2 i-1} \in\{1, r\}, \quad y_{2 i-1}\left|y / d_{y}, \quad u_{2 i-1}^{\prime}\right| u .
\end{gathered}
$$

will be denoted by $C_{2 i-1}$. The set of conditions $C_{2 i}$ are the same as $C_{2 j-1}$ (with $2 j-1$ replaced by $2 i$ ) except that instead of requiring $z_{2 j-1}^{\prime} \mid z$, we need $y_{2 i}^{\prime} \mid y$.

Theorem 5.1. Suppose there exists a complete quotient $\left(\omega_{n}+P_{h_{2 i-1}}\right) / Q_{h_{2 i-1}}$ satisfying

$$
\begin{aligned}
P_{h_{2 i-1}} & =S_{2} / d-s m_{2 i-1} r_{2 i-1} u_{2 i-1} u_{2 i-1}^{\prime} z_{2 i-1} z_{2 i-1}^{\prime} c_{2 i-1} U^{k i} / d \\
Q_{h_{2 i-1}} & =r_{2 i-1} l_{2 i-1} m_{2 i-1}\left(s u_{2 i-1} y_{2 i-1} z_{2 i-1}\right)^{2}(U T)^{k i},
\end{aligned}
$$

where $n>k i$ and the set of conditions $C_{2 i-1}$ are satisfied. Then there is a complete quotient $\left(\omega_{n}+P_{h_{2 i}}\right) / Q_{h_{2 i}}$, where

$$
\begin{aligned}
P_{h_{2 i}} & =S_{1} / d-N s r_{2 i} l_{2 i} u_{2 i} u_{2 i}^{\prime} y_{2 i} y_{2 i}^{\prime} c_{2 i} U^{n-k i} T^{n+k} / d \\
Q_{h_{2 i}} & =r_{2 i} l_{2 i} m_{2 i}\left(s u_{2 i} y_{2 i} z_{2 i}\right)^{2} N T^{n+k(i+1)} U^{n-k i}
\end{aligned}
$$

and the conditions $C_{2 i}$ are satisfied.

Observe that with appropriate selections, $\theta_{h_{1}}$ is a complete quotient satisfying (5-8) and the conditions $C_{1}$.

Proof. From (5-8), we find that the current line in the continued fraction expansion is

$$
\begin{gathered}
\frac{\omega_{n}+P_{h_{2 i-1}}}{Q_{h_{2 i-1}}}=\frac{c q r N x^{n}-s r_{2 i-1} m_{2 i-1} u_{2 i-1} u_{2 i-1}^{\prime} z_{2 i-1} z_{2 i-1}^{\prime} c_{2 i-1} U^{k i}}{d r_{2 i-1} m_{2 i-1} l_{2 i-1}\left(s u_{2 i-1} z_{2 i-1} y_{2 i-1}\right)^{2}(U T)^{k i}}- \\
\frac{\left(\bar{\omega}_{n}+S_{1} / d\right)}{r_{2 i-1} m_{2 i-1} l_{2 i-1}\left(s u_{2 i-1} z_{2 i-1} y_{2 i-1}\right)^{2}(U T)^{k i}}
\end{gathered}
$$

Now, define

$$
\begin{aligned}
& A_{2 i-1}:=\operatorname{cqrNx} x^{n}-s r_{2 i-1} m_{2 i-1} u_{2 i-1} u_{2 i-1}^{\prime} z_{2 i-1} z_{2 i-1}^{\prime} c_{2 i-1} U^{k i}, \\
& B_{2 i-1}:=d r_{2 i-1} m_{2 i-1} l_{2 i-1}\left(s u_{2 i-1} z_{2 i-1} y_{2 i-1}\right)^{2}(U T)^{k i}, \\
& \Delta_{2 i-1}:=\left(A_{2 i-1}, B_{2 i-1}\right) .
\end{aligned}
$$

The next few results aid in determining common factors.

Lemma 5.2. If $A_{2 i-1}=d P_{h_{2 i-1}}+\left(s_{1}+t d\right) / 2$ then $d_{y} u_{2 i-1} l_{2 i-1} y_{2 i-1} \mid A_{2 i-1}$. 
Lemma 5.3. For any rational integers $a, b, d, f$ such that $d \mid a b$ and $(f, d)=1$ there exist rational integers $x, y$ such that

$$
d x y=a b
$$

and $x|a, y| b,(f x, b / y)=1$.

Proof. Take $x=a /(a, d)$ and $y=b(a, d) / d$.

Returning to the expansion of $\omega_{n}$, we write

$$
w_{2 i-1}:=\left(m_{2 i-1}, u / u_{2 i-1}\right) \quad \text { and } \quad e_{2 i-1}:=s d_{y} u_{2 i-1} l_{2 i-1} y_{2 i-1} r_{2 i-1} w_{2 i-1} .
$$

Note that if $n \geq k i$ then $w_{2 i-1} u_{2 i-1} s U^{k i} r_{2 i-1} \mid A_{2 i-1}$ and by Lemma 5.2 we get $d_{y} u_{2 i-1} l_{2 i-1} y_{2 i-1} \mid A_{2 i-1}$. Now we define $G_{2 i-1}:=A_{2 i-1} /\left(U^{k i} e_{2 i-1}\right)$. It follows easily (see [Patterson 2003, Chapter 16]) that $\left(G_{2 i-1}, s T z_{2 i-1} m_{2 i-1} / w_{2 i-1}\right)=1$. In summary,

$$
\Delta_{2 i-1}=U^{k i} e_{2 i-1}\left(G_{2 i-1}, \bar{d} d_{z} y_{2 i-1} u_{2 i-1}\right) .
$$

Since $A_{2 i-1}=d P_{h_{2 i-1}}+S_{1}+t d$, we have $\left(d, A_{2 i-1}\right)=d_{y}$. Thus $\left(\bar{d} d_{z}, G_{2 i-1}\right)=1$. Hence, $\Delta_{2 i-1}=U^{k i} e_{2 i-1} \bar{\Delta}_{2 i-1}$, where $\bar{\Delta}_{2 i-1}:=\left(y_{2 i-1} u_{2 i-1}, G_{2 i-1}\right)$.

From the complete quotient

$$
\theta_{h_{2 i-1}}=\frac{A_{2 i-1}}{B_{2 i-1}}-\frac{\bar{\alpha}}{r_{2 i-1} l_{2 i-1} m_{2 i-1}\left(s u_{2 i-1} y_{2 i-1} z_{2 i-1}\right)^{2}(U T)^{k i}}
$$

we apply Lemma 2.2, so the next partial quotients are those of the expansion of $A_{2 i-1} / B_{2 i-1}$ of length $p_{2 i-1}$. The parity of $p_{2 i-1}$ is determined by $(-1)^{p_{2 i-1}+1}=$ $\operatorname{sign}(l)$. Following this, the next complete quotient is $\theta_{h_{2 i}}$, where $h_{2 i}:=h_{2 i-1}+$ $p_{2 i-1}$. By Lemma 2.2, $\theta_{h_{2 i}}$ is equal to

$$
\begin{aligned}
& \frac{r_{2 i-1} l_{2 i-1} m_{2 i-1}\left(s u_{2 i-1} y_{2 i-1} z_{2 i-1}\right)^{2}(U T)^{k i}}{-\bar{\alpha}\left(B_{2 i-1} / \Delta_{2 i-1}\right)^{2}}-\frac{c_{2 i}}{B_{2 i-1} / \Delta_{2 i-1}}= \\
& \frac{r_{2 i-1} l_{2 i-1}\left(\omega_{n}+S_{1} / d\right)\left(w_{2 i-1} d_{y} \bar{\Delta}_{2 i-1}\right)^{2}}{s^{2} u^{2} r N|l| y^{2} m_{2 i-1} z_{2 i-1}^{2} U^{n-k i} T^{n+k(i+1)}}-\frac{c_{2 i}}{B_{2 i-1} / \Delta_{2 i-1}},
\end{aligned}
$$

where

$$
c_{2 i} \equiv-\operatorname{sign}(l) \Delta_{2 i-1} / A_{2 i-1} \quad\left(\bmod B_{2 i-1} / \Delta_{2 i-1}\right) .
$$

Also note that $s T \mid B_{2 i-1} / \Delta_{2 i-1}$ and $\left(A_{2 i-1} / \Delta_{2 i-1}, B_{2 i-1} / \Delta_{2 i-1}\right)=1$ imply that $\left(c_{2 i}, s T\right)=1$.

According to Lemma 5.3 there exists $y_{2 i}, u_{2 i}$ such that

$$
y_{2 i}\left|y / d_{y}, \quad u_{2 i}\right| u / w_{2 i-1}, \quad y_{2 i} u_{2 i} \bar{\Delta}_{2 i-1}=\frac{y}{d_{y}} \frac{u}{w_{2 i-1}}
$$

and $\left(z_{2 i-1} y_{2 i}, u /\left(u_{2 i} w_{2 i-1}\right)\right)=1$. By taking $l_{2 i}:=|l| / l_{2 i-1}, r_{2 i}:=r / r_{2 i-1}$, $m_{2 i}:=m_{2 i-1}, z_{2 i}:=z_{2 i-1}, y_{2 i}^{\prime}:=y / y_{2 i-1}$, and $u_{2 i}^{\prime}:=u / u_{2 i-1}$, one finds that 
$\left(y_{2 i} z_{2 i}, u / u_{2 i}\right)=1$. Moreover, the complete quotient $\theta_{h_{2 i}}$ now satisfies (5-9) and the set of conditions $C_{2 i}$.

There is also an analogous result for $\theta_{h_{2 i}}$.

Theorem 5.4. Suppose there is a complete quotient $\theta_{h_{2 i}}=\left(\omega_{n}+P_{h_{2 i}}\right) / Q_{h_{2 i}}$ satisfying (5-9) and the set of conditions $C_{2 i}$. Then there is a complete quotient $\theta_{h_{2 i+1}}$, where

$$
\begin{aligned}
P_{h_{2 i+1}} & =S_{2} / d-s m_{2 i+1} r_{2 i+1} u_{2 i+1} u_{2 i+1}^{\prime} z_{2 i+1} z_{2 i+1}^{\prime} c_{2 i+1} U^{k(i+1)} / d \\
Q_{h_{2 i+1}} & =r_{2 i+1} l_{2 i+1} m_{2 i+1}\left(s u_{2 i+1} z_{2 i+1} y_{2 i+1}\right)^{2}(U T)^{k(i+1)}
\end{aligned}
$$

and the set of conditions $C_{2 i+1}$ are satisfied.

Thus, from the complete quotient $\theta_{h_{2 i-1}}$ we find another complete quotient $\theta_{h_{2 i+1}}$ satisfying exactly the same requirements as $\theta_{h_{2 i-1}}$. Moreover, only a bounded number of the these complete quotients are not reduced. More precisely,

$$
\frac{A_{2 i}}{B_{2 i}}=\frac{k_{1} x^{n}-k_{2} U^{n-k i} T^{n+k}}{k_{3} T^{n+k(i+1)} U^{n-k i}}=\frac{k_{1} U^{k i}}{k_{3} T^{k(i+1)}}-\frac{k_{2}}{k_{3} T^{k i}}>1
$$

for some $i \geq W$, where $W$ only depends on the parameters $m, l, y, z, r, d, c, N$, $U, T$.

Similarly,

$$
\frac{A_{2 i+1}}{B_{2 i+1}}=\frac{k_{1} x^{n}-k_{2} U^{k(i+1)}}{k_{3}(U T)^{k(i+1)}}=\frac{k_{1} x^{n}-k_{2}^{\prime} U^{k i}}{k_{3}^{\prime}(U T)^{k i}}=\frac{k_{1} x^{n-k i}}{k_{3}^{\prime}}-\frac{k_{2}^{\prime}}{k_{3}^{\prime} T^{k i}}>1
$$

for some $V$ such that $n-k i \geq V$. Again, $V$ depends only on the parameters $m, l$, $y, z, r, d, c, N, U, T$.

Since the pairs $\left(P_{h_{i}}, Q_{h_{i}}\right)$ are all distinct for $i=1,2, \ldots, 2(n-V) / k+1$, we see that

$$
\operatorname{lp}\left(\omega_{n}\right)>\sum_{j=W}^{(n-V) / k} p_{2 j} .
$$

Our interest now falls on the length of the expansion of $A_{2 i} / B_{2 i}$. Basically, since we have $\Theta(n)$ of these expansions, if the lengths are unbounded then, from above, the period length can not possibly be linear in $n$. In the next section we show that in order to have the length of $A_{2 i} / B_{2 i}$ bounded for all $i$ we must have $T=1$.

\section{The length of the continued fraction of $A_{2 i} / B_{2 i}$}

Let $i$ be fixed with $W \leq i \leq(n-V) / k$, so that $\theta_{h_{2 i}}$ is reduced. We have

$$
P_{h_{2 i}}=S_{1} / d-N s l_{2 i} r_{2 i} u_{2 i} u_{2 i}^{\prime} y_{2 i} y_{2 i}^{\prime} c_{2 i} U^{n-k i} T^{n+k} / d=S_{1} / d-J / d .
$$


As usual, we have

$$
d^{2}\left(t-D_{n}\right) / 4+d^{2}\left(t P_{h_{2 i}}+P_{h_{2 i}}^{2}\right) \equiv 0 \quad\left(\bmod d^{2} Q_{h_{2 i}}\right),
$$

where in the above case

$$
Q_{h_{2 i}}=r_{2 i} m_{2 i} l_{2 i} N\left(s u_{2 i} z_{2 i} y_{2 i}\right)^{2} U^{n-k i} T^{n+k(i+1)} .
$$

We can write this as

$$
-G x^{n}-t d J-2 S_{1} J+J^{2} \equiv 0 \quad\left(\bmod d^{2} Q_{h_{2 i}}\right) .
$$

Modulo $T^{k(i+1)}$ we have

$$
G U^{k i} \equiv-C N s l_{2 i} r_{2 i} u_{2 i} u_{2 i}^{\prime} y_{2 i} y_{2 i}^{\prime} c_{2 i} T^{k}\left(\bmod T^{k(i+1)}\right) .
$$

Returning now to $A_{2 i} / B_{2 i}$, we previously found that

$$
\frac{A_{2 i}}{B_{2 i}}=\frac{A U^{k i}-N s r_{2 i} l_{2 i} u_{2 i} u_{2 i}^{\prime} y_{2 i} y_{2 i}^{\prime} c_{2 i} T^{k}}{d N l_{2 i} r_{2 i} m_{2 i}\left(s u_{2 i} y_{2 i} z_{2 i}\right)^{2} T^{k(i+1)}} .
$$

Writing (6-1) as

$$
\operatorname{CNs}_{2 i} r_{2 i} u_{2 i} u_{2 i}^{\prime} y_{2 i} y_{2 i}^{\prime} c_{2 i} T^{k}=-G U^{k i}-f_{2 i} T^{k(i+1)},
$$

where $f_{2 i} \in \mathbb{Z}$, we find,

$$
\frac{A_{2 i}}{B_{2 i}}=\frac{c^{2} r N m z^{2} U^{k(i+1)}+f_{2 i} T^{k(i+1)}}{C N d l_{2 i} r_{2 i} m_{2 i}\left(s u_{2 i} y_{2 i} z_{2 i}\right)^{2} T^{k(i+1)}}=\frac{1}{F_{2 i}}\left(E \xi^{i+1}+f_{2 i}\right),
$$

where $F_{2 i}$ and $E$ are bounded integers and $\xi=U^{k} / T^{k}$.

Depth of a sequence of rationals. We now provide an aside regarding the depth of $(a / b)^{h}$ as $h \rightarrow \infty$ for coprime integers $a$ and $b$. The depth of the regular continued fraction expansion of $\alpha \in \mathbb{Q}$ is denoted by $\delta(\alpha)$. This is defined as the number of partial quotients in the even length continued fraction expansion of $\alpha$.

Theorem 6.1. If $a$ and $b$ are two coprime integers with $1<b<a$ then

$$
\lim _{i \rightarrow \infty} \delta\left((a / b)^{i}\right)=\infty
$$

Whether this is so was asked by Mendès France and proved by Pourchet in a letter to him. A summary of Pourchet's response is given in [van der Poorten 1984]. ${ }^{3}$

\footnotetext{
${ }^{3}$ Van der Poorten provides the following correction to the given argument: Consider $p_{n+1}<$ $p_{n} a^{h \varepsilon_{n}}$ so that $a^{h}=p_{\psi(h)}<a^{h\left(\varepsilon_{1}+\cdots+\varepsilon_{\psi(h)}\right)}$ and then consider $\varepsilon_{1}+\ldots \varepsilon_{\psi(h)}=\psi(h) \varepsilon$.
} 
It is clear that $\delta(1 / \alpha) \geq \delta(\alpha)-2, \delta(-\alpha) \geq \delta(\alpha)-2$ and $\delta(\alpha+n)=\delta(\alpha)$ for any $n \in \mathbb{Z}$. Furthermore, Mendès France [1973] has shown that for any $\alpha \in \mathbb{Q}, n \in \mathbb{Z}^{+}$,

$$
\delta(n \alpha) \geq \frac{\delta(\alpha)-1}{\kappa(n)+2}-1,
$$

where $\kappa(n)$ is a positive valued function whose values depend only on $n$. Consequently, for any sequence $\alpha_{i} \in \mathbb{Q}$ satisfying $\lim _{i \rightarrow \infty} \delta\left(\alpha_{i}\right)=\infty$ and any $n \in \mathbb{Z}^{+}$we have $\lim _{i \rightarrow \infty} \delta\left(\alpha_{i} / n\right)=\infty$. It follows that, if $T>1$, then

$$
\lim _{i \rightarrow \infty} \delta\left(\frac{A_{2 i}}{B_{2 i}}\right)=\lim _{i \rightarrow \infty} \delta\left(\frac{1}{F_{2 i}}\left(E \xi^{i+1}+f_{2 i}\right)\right)=\infty .
$$

Period length of $\omega_{n}$. By our criteria for a kreeper we must have for some $a, b \in \mathbb{Q}$,

$$
a n+b=l p\left(\omega_{n}\right)>\sum_{j=W}^{(n-V) / k} p_{2 j}
$$

for all $n \in I$. Hence we must have

$$
\sum_{i=W}^{(n-V) / k} \delta\left(\frac{A_{2 i}}{B_{2 i}}\right)<a n+b .
$$

By (6-2) there exists a $\gamma>W$ such that $\delta\left(A_{2 i} / B_{2 i}\right)>k(a+1)$ for all $i \geq \gamma$. Then,

$$
\sum_{i=W}^{(n-V) / k} \delta\left(\frac{A_{2 i}}{B_{2 i}}\right)>k(a+1)[(n-V) / k-\gamma] .
$$

When $n>b+(a+1)(V+k \gamma)$ we have (6-4) is greater than $a n+b$. And since all the terms on the right side of this inequality are bounded, there must exist an infinitude of values of $n \in I$ such that $\operatorname{lp}\left(\omega_{n}\right)>a n+b$ for any fixed $a, b$. In conclusion $D_{n}$ can not be a kreeper if $T>1$. In other words, we must have $T=1$ and $U=x$.

Our only remaining objective is to show that necessarily $N=1$. Clearly, there is no loss in generality in supposing that $N$ is not a power of $x$. In Section 5 we established the existence of the following complete quotient in all kreepers,

$$
\theta_{h_{2 i}}=\frac{\omega_{n}+S_{1} / d-N s r_{2 i} l_{2 i} u_{2 i} u_{2 i}^{\prime} y_{2 i} y_{2 i}^{\prime} c_{2 i} x^{n-k i} / d}{r_{2 i} m_{2 i} l_{2 i}\left(s u_{2 i} y_{2 i} z_{2 i}\right)^{2} N x^{n-k i}} .
$$

By taking $i=\lfloor n / k\rfloor$ we find an element, $\eta \in \mathcal{O}_{n}$, whose norm can be written as $R N x^{v}$, where $R \mid D_{n},\left(N, E_{n}\right)=1$ and $0 \leq v<k$. Hence the norm of $\eta$ is bounded independently of $n$, and coprime to the conductor of the order $O_{n}^{*}$. First, recall the ideal $\mathfrak{b}_{n} \in \mathbb{O}_{n}^{*}$ from page 195 , which has norm $x^{g}$, with $g$ fixed independently of 
$n$. Suppose that $N>1$ and that the ideals $(\eta), \mathfrak{b}_{n}$ are dependent in $\mathbb{O}_{n}^{*}$. Then by Theorem 2.7 we must have $N^{e} x^{v e}=x^{g f}$ for some nonnegative integers $e, f$, not both zero. Since $N$ is not a power of $x$, we must have $e=0, f>0$. But this would imply $x=1$, which is impossible. Hence $(\eta)$ and $\mathfrak{b}_{n}$ are independent ideals in $\mathfrak{O}_{n}^{*}$. Then by Proposition 4.3, $R\left(\mathbb{O}_{n}\right) \gg\left(\log D_{n}\right)^{3}$; that is the sequence of discriminants $\left\{D_{n}\right\}$ is not a kreeper. Thus we must have $N=1$.

This completes the proof of Theorem 1.1.

\section{Function field kreepers}

Almost everything done here is also valid in function fields. Instead of considering quadratic fields over $\mathbb{Q}$, we can consider the so-called congruence quadratic function fields $\mathbb{F}_{q}(X, \sqrt{f})$, where $f \in \mathbb{F}_{q}[X]$. It is well-known that the results in Section 2 have similar analogues for expansions over $\mathbb{F}_{q}[X]$. The main exception for our interests here, is that the continued fraction expansion of a rational function $f(X) \in \mathbb{F}_{q}[X]$ has a fixed length. This was used in Section 5 to ensure each $Q_{h_{j}}$ was positive. In the function field case the term $(-1)^{p_{2 i-1}+1} l$ is equal to $u l$, where $u \in \mathbb{F}_{q}^{*}$. By interpreting $|l|$ to be equal to $u l$ for some $u \in \mathbb{F}_{q}^{*}$, the results carry over.

Other minor details: $P$ lies in $\mathbb{F}_{q}^{*}$ rather than in $\{ \pm 1\}$, which is easily handled by just renaming $M$; further, $L, g$ should be defined to be 2 and $\tau_{y}, \tau_{z}$ can be ignored.

The main problem is that Theorem 6.1 does not hold for coprime functions in $\mathbb{F}_{q}[X]$. Consequently, we have no direct proof that $T=1$ and $U=x$. However, if we suppose only the weaker condition: that $x$ is a monomial in $X$ (then necessarily $x=X$ by assumption about powers of $x$ ) then trivially, $T \in \mathbb{F}_{q}$ and $U=x / T$. Then by renaming $l$ and $m$ we have:

Theorem 7.1. A function field kreeper, that is a sequence of polynomials $f_{n}(X)$ such that

(1) $f_{n}(X)=A(X)^{2} X^{2 n}+B(X) X^{n}+C^{2}$, where $A, B, C \in \mathbb{F}_{q}[X]$

(2) $\operatorname{lp}\left(\sqrt{f_{n}(X)}\right)=a n+b$ for some $a, b \in \mathbb{Q}$

(3) In the principal cycle there exists an element whose norm is $X^{g}$ for some $g$ fixed independently of $n$.

must satisfy

$$
d^{2} f_{n}(X)=c^{2}\left(\left(q r X^{n}+\left(m z^{2} X^{k}-l y^{2}\right) / q\right)^{2}+4 r l y^{2} X^{n}\right),
$$

where $q, r, l, m \in \mathbb{F}_{q}[X]$ and

$$
\begin{gathered}
(q r X, m l z y)=1, \quad(m l, z y)=1, \quad(q r, X)=1, \\
c^{2} r l y^{2} m z^{2}\left|d^{2} D_{n}, \quad q\right| m z^{2} X^{k}-l y^{2} .
\end{gathered}
$$




\section{Some more notations}

Define $s:=\max _{i \in \mathbb{N}}\left\{\left(x^{i}, c\right)\right\}, u:=c / s$ so that $(u, x)=1$. In order to make things easier for ourselves when we come to the expansion of $\omega_{n}$, we would like to have

$$
x^{\mu} \equiv 1 \quad\left(\bmod u^{2} m z^{2} l y^{2}\right) \quad \text { and } \quad x^{\mu} \equiv 0 \quad\left(\bmod s^{2}\right) .
$$

Clearly, such a $\mu$ must exist. We shall want to consider the congruence class, $I_{\nu}:=\{n \in \mathbb{N}: n \equiv v(\bmod \mu), n>\mu\}$. Our proof will show that $D_{n}$ is a kreeper for $n \in I_{\nu}$, and since every $n$ lies in some $I_{\nu}$, we shall not be losing any generality in this restriction. Moreover, the value of $x^{n}\left(\bmod u^{2} s^{2} m z^{2} l y^{2}\right)$ is the same for all $n \in I_{v}$.

If $\theta_{i+1}$ represents the $(i+1)$-th complete quotient of $\omega_{n}$, that is, if

$$
\omega_{n}=\left[a_{0}, a_{1}, \ldots, a_{i}, \theta_{i+1}\right],
$$

we define

$$
\Psi_{i+1}=\theta_{1} \ldots \theta_{i-1} \theta_{i} Q_{i} \in \mathbb{Z}\left[\omega_{n}\right] .
$$

Then, we have $N\left(\Psi_{i+1}\right)=(-1)^{i} Q_{i}$. If we write the complete quotient $\theta_{h_{i}}=$ $\left(\omega_{n}+P_{h_{i}}\right) / Q_{h_{i}}$ as $\theta_{h_{i}}=A_{i} / B_{i}-\gamma$, where we take

$$
B_{i}=d Q_{h_{i}} \quad \text { and } \quad A_{i}= \begin{cases}d P_{h_{i}}+c q r x^{n}-S_{m} & \varepsilon_{i}=0 \text { or } i \equiv 0 \quad(\bmod 2), \\ d P_{h_{i}}+s_{1}-S_{2} & \varepsilon_{i-1}=1 \text { and } i \equiv 1 \quad(\bmod 2),\end{cases}
$$

where $P_{h_{i}}=S_{m} / d-J, S_{m}$ being one of $S_{1}$ and $S_{2}$ and $J$ is some function of $r, l$, $m, z, y, c, x$. Applying Lemma $2.2\left(\right.$ where $\Delta_{i}=\left(A_{i}, B_{i}\right)$ ), we find

$$
\theta_{h_{i+1}}=\frac{(-1)^{p_{i}} \bar{\gamma}}{\gamma \bar{\gamma}\left(B_{i} / \Delta_{i}\right)^{2}}-\frac{c_{i}}{B_{i} / \Delta_{i}}
$$

where $c_{i} \equiv(-1)^{p_{i}+1} \Delta_{i} / A_{i}\left(\bmod B_{i} / \Delta_{i}\right)$. Thus,

$$
Q_{h_{i+1}}=(-1)^{p_{i}} \gamma \bar{\gamma}\left(B_{i} / \Delta_{i}\right)^{2} Q_{h_{i}} .
$$

Hence,

$$
\frac{(-1)^{p_{i}} Q_{h_{i+1}}}{Q_{h_{i}}}=\frac{\left(A_{i}-B_{i} \theta_{h_{i}}\right)\left(A_{i}-B_{i} \bar{\theta}_{h_{i}}\right)}{\Delta_{i}^{2}} .
$$

By Corollary 2.4,

$$
\theta_{h_{i}+1} \theta_{h_{i}+2} \ldots \theta_{h_{i+1}}=\frac{\left(A_{i}-\bar{\theta}_{h_{i}} B_{i}\right) Q_{h_{i}}}{\Delta_{i} Q_{h_{i+1}}} .
$$

Hence,

$$
\Psi_{h_{i+1}+1}=\left(\frac{A_{i}-B_{i} \bar{\theta}_{h_{i}}}{\Delta_{i}}\right) \Psi_{h_{i}+1}
$$




\section{Determination of some specific elements in the expansion}

In Section 5 we determined the existence of the following complete quotient in the expansion of $\omega_{n}$,

$$
\theta_{h_{1}}=\frac{\omega_{n}+S_{2} / d-c_{1}(c / \delta) z\left(z / d_{z}\right) x^{k} / d}{(c / \delta)^{2}|m l|\left(y / d_{y}\right)^{2}\left(z / d_{z}\right)^{2} x^{k}}
$$

Moreover, for sufficiently large $n, \theta_{h_{1}}$ is reduced. Furthermore,

$$
\Psi_{h_{1}+1}=\alpha \beta /\left(d \Delta_{0}\right) .
$$

As shown earlier, the development of the expansion of $\omega_{n}$ depends upon whether or not a power of $x^{k}$ can be factored out from $Q_{h_{i}}$ or not. In order to accommodate this we define

$$
\varepsilon_{i}= \begin{cases}1 & \text { if } \lambda_{i} \geq n-k \\ 0 & \text { if } \lambda_{i}<n-k\end{cases}
$$

where $\lambda_{i}$ is defined recursively as

$$
\lambda_{1}:=k, \quad \lambda_{i+2}:=\left\{\begin{array}{lll}
\lambda_{i}+k-n \varepsilon_{i} & \text { if } i \equiv 1 & (\bmod 2), \\
0 & \text { if } i \equiv 0 & (\bmod 2) .
\end{array}\right.
$$

Note that $\lambda_{2 i-1} \equiv k i(\bmod n)$.

Theorem 9.1. Suppose there exists a complete quotient $\left(\omega_{n}+P_{h_{i}}\right) / Q_{h_{i}}$ satisfying

$$
P_{h_{i}}=S_{2} / d-s m_{i} r_{i} u_{i} u_{i}^{\prime} z_{i} z_{i}^{\prime} c_{i} x^{\lambda_{i}} / d, \quad Q_{h_{i}}=r_{i} l_{i} m_{i}\left(s u_{i} y_{i} z_{i}\right)^{2} x^{\lambda_{i}},
$$

and the set of conditions $C_{i}$ are satisfied. Then there exists a complete quotient $\left(\omega_{n}+P_{h_{i+2}}\right) / Q_{h_{i+2}}$ satisfying (9-3) and $C_{i+2}$. Furthermore,

$$
\Psi_{h_{i+2}+1}=\left(\frac{\alpha^{\varepsilon_{i}+1} \beta}{d^{2} \Delta_{i} \Delta_{i+1}}\right) \Psi_{h_{i}+1}
$$

Note: It is clear that with the appropriate selections, the complete quotient $\theta_{h_{1}}$ in (9-1) satisfies the conditions of the theorem.

Proof. In Section 5 we determined the existence of a complete quotient satisfying the conditions $C_{i+1}^{\prime}$. Furthermore,

$$
\Psi_{h_{i+1}+1}=\left(\frac{A_{i}-B_{i} \bar{\theta}_{h_{i}}}{\Delta_{i}}\right) \Psi_{h_{i}+1}=\left(\frac{\alpha}{d \Delta_{i}}\right) \Psi_{h_{i}+1} .
$$

Moreover we have $h_{i+1}=h_{i}+p_{i}$, where $p_{i}$ is the length of the appropriately selected continued fraction expansion of $A_{i} / B_{i}$.

Suppose $\varepsilon_{i}=0$. If $\lambda_{i}<n-k$ then $\varepsilon_{i}=0$ and $\lambda_{i+2}=\lambda_{i}+k$. This now follows as in Section 5. 
Suppose $\varepsilon_{i}=1$. When $\lambda_{i} \geq n-k$ we have $\varepsilon_{i}=1$ and this situation has not yet been considered. In this situation, the previous choice of $A_{i+1}$ is not appropriate. Instead, we need to consider

$$
\begin{array}{r}
\theta_{h_{i+1}}=\frac{s_{1} / d-s r_{i+1} l_{i+1} u_{i+1} u_{i+1}^{\prime} y_{i+1} y_{i+1}^{\prime} c_{i+1} x^{n-\lambda_{i}} / d}{r_{i+1} l_{i+1} m_{i+1}\left(s u_{i+1} y_{i+1} z_{i+1}\right)^{2} x^{n-\lambda_{i}}}- \\
\frac{\bar{\alpha}}{r_{i+1} l_{i+1} m_{i+1}\left(s u_{i+1} y_{i+1} z_{i+1}\right)^{2} x^{n-\lambda_{i}}} .
\end{array}
$$

There is a slight problem in notation because there is going to be an extra intermediate complete quotient. Consequently, we will use overlines to represent the terms involved. This time we take

$$
\begin{aligned}
& \bar{A}_{i+1}:=s_{1}-s r_{i+1} l_{i+1} u_{i+1} u_{i+1}^{\prime} y_{i+1} y_{i+1}^{\prime} c_{i+1} x^{n-\lambda_{i}}, \\
& \bar{B}_{i+1}:=d r_{i+1} l_{i+1} m_{i+1}\left(s u_{i+1} y_{i+1} z_{i+1}\right)^{2} x^{n-\lambda_{i}}, \\
& \bar{\Delta}_{i+1}:=\left(\bar{A}_{i+1}, \bar{B}_{i+1}\right) .
\end{aligned}
$$

Then, $\bar{A}_{i+1}=d P_{h_{i+1}}+\left(s_{1}+t d\right) / 2$, which leads to $d_{y} u_{i+1} l_{i+1} y_{i+1} \mid \bar{A}_{i+1}$ after a short calculation. We also define

$$
\bar{w}_{i+1}:=\left(m_{i+1}, u / u_{i+1}\right), \quad \bar{e}_{i+1}:=s d_{y} u_{i+1} l_{i+1} y_{i+1} r_{i+1} \bar{w}_{i+1} .
$$

By writing $\Delta_{i+1}^{\prime}=\left(\bar{G}_{i+1}, u_{i+1} y_{i+1}\right)$, a little calculation gives

$$
\bar{\Delta}_{i+1}=s d_{y} u_{i+1} l_{i+1} y_{i+1} r_{i+1} \bar{w}_{i+1} \Delta_{i+1}^{\prime} .
$$

Note that

$$
\frac{\bar{B}_{i+1}}{\bar{\Delta}_{i+1}}=\frac{s d m_{i+1} u_{i+1} y_{i+1} z_{i+1}^{2} x^{n-\lambda_{i}}}{d_{y} \bar{w}_{i+1} \Delta_{i+1}^{\prime}} .
$$

According to Lemma 5.3 there exists two numbers $\bar{y}_{i+2}$ and $\bar{u}_{i+2}$, such that

$$
\bar{y}_{i+2}\left|y / d_{y}, \quad \bar{u}_{i+2}\right| u / \bar{w}_{i+1}, \quad \bar{y}_{i+2} \bar{u}_{i+2} \Delta_{i+1}^{\prime}=\frac{y}{d_{y}} \frac{u}{\bar{w}_{i+1}},
$$

and

$$
\left(z_{i+1} \bar{y}_{i+2}, \frac{u}{\bar{u}_{i+2} \bar{w}_{i+1}}\right)=1 \text {. }
$$

From

$$
\theta_{h_{i+1}}=\frac{\bar{A}_{i+1}}{\bar{B}_{i+1}}-\frac{\bar{\alpha}}{r_{i+1} l_{i+1} m_{i+1}\left(s u_{i+1} y_{i+1} z_{i+1}\right)^{2} x^{n-\lambda_{i}}},
$$

we apply Lemma 2.2 and find that the next partial quotients are those of the continued fraction expansion of $\bar{A}_{i+1} / \bar{B}_{i+1}$ of length $\bar{p}_{i+1}$, where the parity of $\bar{p}_{i+1}$ 
is determined by $(-1)^{\bar{p}_{i+1}+1}=\operatorname{sign}(l)$. The next complete quotient is then $\theta_{j_{i+2}}$, where $j_{i+2}=h_{i+1}+\bar{p}_{i+1}$ and

$$
\theta_{j_{i+2}}=\frac{r_{i+1} l_{i+1} m_{i+1}\left(s u_{i+1} y_{i+1} z_{i+1}\right)^{2} x^{n-\lambda_{i}}}{-\operatorname{sign}(l) \bar{\alpha}\left(\frac{s d m_{i+1} u_{i+1} y_{i+1} z_{i+1}^{2} x^{n-\lambda_{i}}}{d_{y} \bar{w}_{i+1} \Delta_{i+1}^{\prime}}\right)^{2}}-\frac{\bar{c}_{i+2}}{\bar{B}_{i+1} / \bar{\Delta}_{i+1}},
$$

where $\bar{c}_{i+2} \equiv-\operatorname{sign}(l) \bar{\Delta}_{i+1} / \bar{A}_{i+1}\left(\bmod \bar{B}_{i+1} / \bar{\Delta}_{i+1}\right)$. The standard choices yield

$$
\theta_{j_{i+2}}=\frac{\omega_{n}+S_{1} / d-s \bar{r}_{i+2} \bar{l}_{i+2} \bar{u}_{i+2} \bar{u}_{i+2}^{\prime} \bar{y}_{i+2} \bar{y}_{i+2}^{\prime} \bar{c}_{i+2} x^{2 n-\lambda_{i}} / d}{\bar{r}_{i+2} \bar{l}_{i+2} \bar{m}_{i+2}\left(s \bar{u}_{i+2} \bar{y}_{i+2} \bar{z}_{i+2}\right)^{2} x^{2 n-\lambda_{i}}}
$$

Since $s \mid \bar{B}_{i+1} / \bar{\Delta}_{i+1}$ we get $\left(\bar{c}_{i+2}, s\right)=1$, also $\left(\bar{z}_{i+2} \bar{y}_{i+2}, u / \bar{u}_{i+2}\right)=1$. Hence, the conditions $\bar{C}_{i+2}^{\prime}$ are satisfied. Furthermore,

$$
\Psi_{j_{i+2}+1}=\left(\frac{\alpha}{d \bar{\Delta}_{i+1}}\right) \Psi_{h_{i+1}+1} .
$$

The complete quotient in (9-6) satisfies the conditions of Theorem 5.4 Hence, there exists a complete quotient

$$
\theta_{j_{i+3}}=\frac{\omega_{n}+S_{2} / d-s \bar{m}_{i+3} \bar{r}_{i+3} \bar{u}_{i+3} \bar{u}_{i+3}^{\prime} \bar{z}_{i+3} \bar{z}_{i+3}^{\prime} \bar{c}_{i+3} x^{\lambda_{i}+k-n} / d}{\bar{r}_{i+3} \bar{l}_{i+3} \bar{m}_{i+3}\left(s \bar{u}_{i+3} \bar{y}_{i+3} \bar{z}_{i+3}\right)^{2} x^{\lambda_{i}+k-n}}
$$

with the conditions $\bar{C}_{i+3}$ satisfied, and

$$
\Psi_{j_{i+3}+1}=\left(\frac{\beta}{d \bar{\Delta}_{i+2}}\right) \Psi_{j_{i+2}+1}=\left(\frac{\alpha \beta}{d^{2} \bar{\Delta}_{i+1} \bar{\Delta}_{i+2}}\right) \Psi_{h_{i+1}+1} .
$$

Since $\varepsilon_{i}=1$ we have $\lambda_{i+2}=\lambda_{i}+k-n$. With appropriate renaming, the complete quotient $\theta_{j_{i+3}}$ in (9-7) becomes

$$
\theta_{h_{i+2}}=\frac{\omega_{n}+S_{2} / d-s r_{i+2} m_{i+2} u_{i+2} u_{i+2}^{\prime} z_{i+2} z_{i+2}^{\prime} c_{i+2} x^{\lambda_{i+2}} / d}{r_{i+2} l_{i+2} m_{i+2}\left(s u_{i+2} y_{i+2} z_{i+2}\right)^{2} x^{\lambda_{i+2}}}
$$

with the conditions $C_{i+2}$ being satisfied. Combining both the $\varepsilon_{i}=0$ and $\varepsilon_{i}=1$ cases, we have

$$
\Psi_{h_{i+2}+1}=\left(\frac{\alpha^{\varepsilon_{i}} \beta}{d \Delta_{i+1}}\right) \Psi_{h_{i+1}+1}=\left(\frac{\alpha^{\varepsilon_{i}+1} \beta}{d^{2} \Delta_{i} \Delta_{i+1}}\right) \Psi_{h_{i}+1},
$$

and $h_{i+2}=h_{i}+p_{i}+p_{i+1}$.

Our next step will be to investigate the number of partial quotients in the period of the continued fraction expansion of $\omega_{n}$. 


\section{Determining the number of partial quotients}

By defining, $f_{0}:=h_{0}$ and $f_{i+1}:=p_{i}$ for $i \geq 1$ we find

$$
h_{i}=\sum_{k=0}^{i} f_{k} \text {. }
$$

The value of $f_{i}$ is dependent only upon the set

$$
Z_{i}:=\left\{l_{i}, m_{i}, r_{i}, s_{i}, s_{i}^{\prime}, z_{i}, z_{i}^{\prime}, u_{i}, u_{i}^{\prime}, y_{i}, y_{i}^{\prime}, c_{i}, L_{i}, x\right\},
$$

where $L_{i}:=\lambda_{i}(\bmod \mu)$. Moreover, if $Z_{i}=Z_{j}$ then $f_{i}=f_{j}$. There are only finitely many distinct sets $Z_{i}$; we denote the total number of distinct sets by $Z$. For a fixed $t$, there are precisely $t k$ values of $i \in\{1, \ldots, 2 t n-1\}$, where $\varepsilon_{i}=1$. Let $i_{1}, \ldots, i_{t k}$ represent these points. Then

$$
i_{h}= \begin{cases}\frac{2 n h}{k}-3 & \text { if } k \mid 2 n h, \\ \left\lfloor\frac{2 n h}{k}\right\rfloor-2 & \text { if } k \backslash 2 n h .\end{cases}
$$

From (10-1),

$$
h_{2 n t-1}=\sum_{i=0}^{i_{1}-1} f_{i}+\sum_{h=1}^{t k-1} f_{i_{h}}+\sum_{h=1}^{t k-1} \sum_{i=i_{h}+1}^{i_{h+1}-1} f_{i} .
$$

The number of summands in $\sum_{i_{h}+1}^{i_{h+1}-1} f_{i}$ is $i_{h+1}-1-\left(i_{h}+1\right)+1 \geq\lfloor 2 n / k\rfloor-2$. Hence the distance between $i_{h}$ and $i_{h+1}$ can be made arbitrarily large. But $Z$ is independent of $n$, which by the box principle means that for large enough $n$, there exists $\rho_{h}$ and $\tau_{h}$ such that

$$
Z_{i_{h}+\tau_{h}}=Z_{i_{h}+\tau_{h}+\rho_{h}} \quad i_{h}+\tau_{h}+\rho_{h} \leq i_{h+1}-1 \quad 1 \leq \tau_{h}, \rho_{h} \leq Z .
$$

Now we examine $Z_{\kappa+i_{h}+\tau_{h}+j \rho_{h}}$, where $\kappa+i_{h}+\tau_{h}+j \rho_{h} \leq i_{h+1}-1$ and $0 \leq \kappa<\rho_{h}$. Since $\varepsilon_{i_{h}+\tau_{h}}=0$ and $\varepsilon_{i_{h}+\tau_{h}+\rho_{h}}=0$ we have

$$
Z_{i_{h}+\tau_{h}+1}=Z_{i_{h}+\tau_{h}+\rho_{h}+1} \quad \text { provided } i_{h}+\tau_{h}+\rho_{h}+1 \leq i_{h+1}-1 .
$$

By induction,

$$
Z_{i_{h}+\tau_{h}+\kappa}=Z_{i_{h}+\tau_{h}+\kappa+j \rho_{h}} \quad \text { provided } i_{h}+\tau_{h}+\kappa+j \rho_{h} \leq i_{h+1}-1,
$$

which implies $f_{i_{h}+\tau_{h}+\kappa}=f_{i_{h}+\tau_{h}+\kappa+j \rho_{h}}$. Define

$$
v:=\left\lfloor\frac{\lfloor 2 n / k\rfloor-\tau_{h}-\rho_{h}}{\rho_{h}}\right\rfloor-1
$$


It is straightforward that $i_{h}+\tau_{h}+\kappa+v \rho_{h}<i_{h+1}$. Then

$$
\sum_{i=i_{h}+1}^{i_{h+1}-1} f_{i}=\sum_{j=1}^{\tau_{h}-1} f_{i_{h}+j}+\sum_{\kappa=0}^{\rho_{h}-1}\left(\sum_{j=0}^{v} f_{i_{h}+\tau_{h}+\kappa+j \rho_{h}}\right)+\sum_{j=i_{h}+\tau_{h}+\rho_{h}+v \rho_{h}}^{i_{h+1}-1} f_{j} .
$$

The number of terms in $\sum_{j=i_{h}+\tau_{h}+\rho_{h}+v \rho_{h}}^{i_{h+1}-1} f_{j}$ is $\leq \rho_{h}+2$. Hence,

$$
\sum_{i=i_{h}+1}^{i_{h+1}-1} f_{i}=\left\lfloor\frac{2 n-k \tau_{h}-k \rho_{h}}{k \rho_{h}}\right\rfloor \zeta_{h}+\xi_{h}
$$

where $\zeta_{h}, \xi_{h}$ are independent of $n$.

We now take $\rho=\prod_{i=1}^{t k} \rho_{h}$ and $w=\operatorname{lcm}[k, \mu, \rho]$ both of which are independent of $n$. We write $n=w \gamma+\phi$, where $0 \leq \phi<w$. From the original set $I_{\nu}$, we now wish to consider the following subset, $I_{v, \phi}=\left\{n \in I_{v}: n \equiv \phi(\bmod w)\right\}$. Without loss of generality, we may suppose that $n \in I_{\nu, \phi}$. Consequently,

$$
\left\lfloor\frac{2 n-k \tau_{h}-k \rho_{h}}{k \rho_{h}}\right\rfloor=2 \gamma \frac{w}{k \rho_{h}}+\left\lfloor\frac{2 \phi-k \tau_{h}-k \rho_{h}}{k \rho_{h}}\right\rfloor .
$$

Thus, the sum (10-2) becomes,

$$
\sum_{i=i_{h}+1}^{i_{h+1}-1} f_{i}=2 \zeta_{h} \gamma \frac{w}{k \rho_{h}}+\zeta_{h}\left\lfloor\frac{2 \phi-k \tau_{h}-k \rho_{h}}{k \rho_{h}}\right\rfloor+\xi_{h},
$$

which means we can now write $h_{2 n t-1}$ as

$$
h_{2 n t-1}=\sum_{i=0}^{i_{1}-1} f_{i}+\sum_{h=1}^{t k-1} f_{i_{h}}+2 \gamma \sum_{h=1}^{t k-1} \zeta_{h} \frac{w}{k \rho_{h}}+\sum_{h=1}^{t k-1}\left(\zeta_{h}\left\lfloor\frac{2 \phi-k \tau_{h}-k \rho_{h}}{k \rho_{h}}\right\rfloor+\xi_{h}\right) .
$$

Now, let

$$
\begin{aligned}
& x_{t}=2 \sum_{h=1}^{t k-1} \zeta_{h} \frac{w}{k \rho_{h}} \\
& y_{t}=\sum_{h=1}^{t k-1}\left(\zeta_{h}\left\lfloor\frac{2 \phi-k \tau_{h}-k \rho_{h}}{k \rho_{h}}\right\rfloor+\xi_{h}\right)+\sum_{i=0}^{i_{1}-1} f_{i}+\sum_{h=1}^{t k-1} f_{i_{h}},
\end{aligned}
$$

both of which are integers and independent of $\gamma$. Then

$$
h_{2 n t-1}=\gamma x_{t}+y_{t}=((n-\phi) / w) x_{t}+y_{t}=a_{t} n+b_{t},
$$

where $a_{t}, b_{t}$ are both rational numbers independent of $n$ for all $n \in I_{\nu, \phi}$. 
This shows that the length of the expansion up to $h_{2 n t-1}$ is linear in $n$. It remains to show that there exists some $h_{2 n t-1}$, where $Q_{h_{2 n t-1}}=1$, and if $Q_{j}=1$ then $j=h_{2 n t-1}$ for some $t$ independent of $n$.

\section{Finding an element of norm 1}

We now examine the product of the elements in the expansion. By Theorem 9.1 and (9-2),

$$
\Psi_{h_{2 i-1}+1}=\frac{(\alpha \beta)^{i} \alpha^{\sum_{j=1}^{i-1} \varepsilon_{2 j-1}}}{d^{2 i-1} \prod_{j=0}^{2 i-2} \Delta_{j}}
$$

So,

$$
\Psi_{h_{2 n t-1}+1}=\frac{(\alpha \beta)^{n t} \alpha^{k t}}{d^{2 n t-1} \prod_{j=0}^{2 n t-2} \Delta_{j}} \quad \text { and } \quad\left|N\left(\Psi_{h_{2 n t-1}+1}\right)\right|=Q_{h_{2 n t-1}} .
$$

Hence,

$$
Q_{h_{2 n t-1}}\left(d^{2 n t-1} \prod_{j=0}^{2 n t-2} \Delta_{j}\right)^{2}=\left|N\left((\alpha \beta)^{n t}\right) N\left(\alpha^{k t}\right)\right|=\left|N\left((\alpha \beta)^{n}\right) N\left(\alpha^{k}\right)\right|^{t}
$$

as well as

$$
Q_{h_{2 n-1}}\left(d^{2 n-1} \prod_{j=0}^{2 n-2} \Delta_{j}\right)^{2}=\left|N\left((\alpha \beta)^{n}\right) N\left(\alpha^{k}\right)\right|
$$

Thus, $\sqrt{Q_{h_{2 n t-1}} /\left(Q_{h_{2 n-1}}\right)^{t}} \in \mathbb{Q}$. Since $\lambda_{2 j-1} \equiv j k(\bmod n)$ we have that $\lambda_{2 n i-1}=0$ for positive $i$. Hence, $\lambda_{2 n-1}=\lambda_{2 n t-1}=0$ and so

$$
\begin{aligned}
Q_{h_{2 n-1}} & =l_{2 n-1} m_{2 n-1} r_{2 n-1}\left(s u_{2 n-1} z_{2 n-1} y_{2 n-1}\right)^{2}, \\
Q_{h_{2 n t-1}} & =l_{2 n t-1} m_{2 n t-1} r_{2 n t-1}\left(s u_{2 n t-1} z_{2 n t-1} y_{2 n t-1}\right)^{2} .
\end{aligned}
$$

Thus,

$$
\sqrt{\frac{l_{2 n t-1} m_{2 n t-1} r_{2 n t-1}}{\left(l_{2 n-1} m_{2 n-1} r_{2 n-1}\right)^{t}}} \in \mathbb{Q} .
$$

Since $l_{2 n-1}, m_{2 n-1}, r_{2 n-1}$ are each squarefree and relatively prime, if $2 \mid t$ then $\sqrt{l_{2 n t-1} m_{2 n t-1} r_{2 n t-1}} \in \mathbb{Q}$, which implies $l_{2 n t-1} m_{2 n t-1} r_{2 n t-1}=1$. Conversely, if $2 \nmid t$, then $l_{2 n t-1} m_{2 n t-1} r_{2 n t-1}=l_{2 n-1} m_{2 n-1} r_{2 n-1}$.

Now, we construct an element of norm 1. Put

$$
\varepsilon= \begin{cases}1 & \text { if } l_{2 n-1} m_{2 n-1} r_{2 n-1} \neq 1 \\ 0 & \text { if } l_{2 n-1} m_{2 n-1} r_{2 n-1}=1\end{cases}
$$


and

$$
\Gamma=\frac{\Psi_{h_{2 n-1}+1}^{1+\varepsilon}}{\left(l_{2 n-1} m_{2 n-1} r_{2 n-1}\right)^{\varepsilon}\left(s u_{2 n-1} y_{2 n-1} z_{2 n-1}\right)^{1+\varepsilon}} .
$$

Then it is easily shown that $N(\Gamma)=1$.

Lemma 11.1. If $D_{n}=F_{n}^{2} D_{n}^{\prime}$, where $D_{n}^{\prime}$ is squarefree, then $s u_{i} y_{i} z_{i} \mid F_{n}$.

The lemma and above results imply that we have $V_{1}, Y_{1} \in \mathbb{Z}$ such that

$$
\Gamma=V_{1}+Y_{1} \frac{F_{n}}{s u_{2 n-1} y_{2 n-1} z_{2 n-1}} \omega_{n}^{\prime},
$$

where $\mathbb{Z}\left[\omega_{n}^{\prime}\right]$ is the maximal order of $\mathbb{Q}\left(\sqrt{D_{n}^{\prime}}\right)$ and $D_{n}^{\prime}$ is the squarefree kernel of $D_{n}$, that is $D_{n}=F_{n}^{2} D_{n}^{\prime}$. If we now define $V_{j}$ and $Y_{j}$ by

$$
\left(V_{j}+Y_{j} \frac{F_{n}}{s u_{2 n-1} y_{2 n-1} z_{2 n-1}} \omega_{n}^{\prime}\right)=\Gamma^{j} .
$$

Then $Y_{j} / Y_{1}$ is the Lucas function, $\left(\Gamma^{j}-\bar{\Gamma}^{j}\right) /(\Gamma-\bar{\Gamma})$. Since $\Gamma \bar{\Gamma}=1$, there must exist some minimal positive $p$ such that $s u_{2 n-1} y_{2 n-1} z_{2 n-1} \mid Y_{p}$. Putting $t=g:=$ $(1+\varepsilon) p$ we get

$$
\Psi_{h_{2 n g-1}+1}=\Gamma^{p} \sqrt{l_{2 n g-1} m_{2 n g-1} r_{2 n g-1}} s u_{2 n g-1} y_{2 n g-1} z_{2 n g-1} .
$$

If $\varepsilon=1$ then $2 \mid g$ implies that $l_{2 n g-1} m_{2 n g-1} r_{2 n g-1}=1$. If $\varepsilon=0$ then

$$
l_{2 n-1} m_{2 n-1} r_{2 n-1}=1,
$$

so $l_{2 n g-1} m_{2 n g-1} r_{2 n g-1}=1$. Since $\Gamma^{p} \in \mathbb{Z}\left[\omega_{n}\right]$, this implies $s u_{2 n g-1} y_{2 n g-1} z_{2 n g-1}=1$. Hence $\left|N\left(\Psi_{h_{2 n g-1}+1}\right)\right|=1$, which means that $Q_{h_{2 n g-1}}=1$. The values of $p$ depend only on $s u_{2 n-1} y_{2 n-1} z_{2 n-1}$, which divides $s u y z$. Thus, there can only be a finite number of possible values for $p$.

Conversely, one can also show (see [Patterson 2003, Chapter 25]) that such a solution is either fundamental or the square of the fundamental solution, although this is superfluous in showing that $D_{n}$ is a kreeper.

\section{Returning to the regular continued fraction expansion}

Up to now we have determined

$$
\omega_{n}=\left[a_{0}, \ldots, a_{h_{0}-1}, b_{1}, a_{h_{0}+1}, \ldots, a_{h_{1}-1}, b_{2}, \ldots, a_{h_{2 n t-1}-1}, 2 a_{0}-t\right],
$$

but in this evaluation we never insisted that $b_{i} \geq 1$. In other words this expansion might not correspond to the regular continued fraction expansion of $\omega_{n}$. This is equivalent to saying that the expansions of $A_{i} / B_{i}$ might have an initial nonpositive partial quotient. 
Proposition 12.1. In the expansion of $\omega_{n}$ given by the earlier procedure, the number of nonpositive partial quotients is bounded independently of $n$.

Proof. See [Patterson 2003].

This proposition means that there are only finitely many partial quotients that need to be altered in order to find the regular continued fraction expansion of $\omega_{n}$.

The removal of nonpositive partial quotients is covered in [Dirichlet 1999]. There it is shown that any negative partial quotient can be moved to the left in the continued fraction expansion. In our situation, we discover that either the negative partial quotient disappears easily or we are left with

$$
\left[b_{i-1},-1,1, u, v, \ldots\right]=\left[b_{i-1}-2-u, 1, v-1, \ldots\right],
$$

where $u>0$ and is bounded independently of $n$, and $b_{i-1}=\left\lfloor A_{i-1} / B_{i-1}\right\rfloor$. When

$$
\theta_{h_{i-1}}=\frac{\omega_{n}+S_{2} / d-E_{i-1} x^{\lambda_{i}} / d}{E_{i-1}^{\prime} x^{\lambda_{i}}}=\frac{A_{i-1}}{B_{i-1}}+e
$$

we have $\lambda_{i} \ll 1$, implies $\left\lfloor A_{i-1} / B_{i-1}\right\rfloor>x^{n-J_{1}}$, where $J_{1}$ is bounded independently of $n$. Consequently, $b_{i-1}-2-u>0$ for all sufficiently large $n$. The other case follows similarly.

Finally, we note that $a_{h_{i}+j}$ with $j>0$ can not be the end of the period since $Q_{h_{i}+j}>1$. For sufficiently large $n$, each $b_{j}$ which is not some $b_{h_{2 n t-1}}$ satisfies $b_{j}<x^{n}$ since $B_{i} \geq x$.

In conclusion, we have shown that,

$$
l p\left(\omega_{n}\right)=h_{2 n g-1}+c_{n}=a_{g} n+b_{g}+c_{n}=a_{g} n+b_{g}^{\prime},
$$

where $c_{n} \in \mathbb{Z}$ can be bounded independently of $n$. Then $a_{g}, b_{g}^{\prime}$ are rational numbers bounded independently of $n$.

Hence there must exist an infinitude of values of $n \in I$ such that

$$
\operatorname{lp}\left(\omega_{n}\right)=a n+b,
$$

where $a, b \in \mathbb{Q}$ and are fixed independently of $n$. This completes the proof of Theorem 1.2.

\section{References}

[Adam 1995] B. Adam, "Voronoŭ-algorithm expansion of two families with period length going to infinity”, Math. Comp. 64:212 (1995), 1687-1704. MR 96a:11113 Zbl 0858.11070

[Adam 1998] B. Adam, “Généralisation d'une famille de Shanks", Acta Arith. 84:1 (1998), 43-58. MR 99d:11115 Zbl 0899.11066

[Azuhata 1984] T. Azuhata, "On the fundamental units and the class numbers of real quadratic fields”, Nagoya Math. J. 95 (1984), 125-135. MR 86b:11069 Zbl 0533.12008 
[Azuhata 1987] T. Azuhata, "On the fundamental units and the class numbers of real quadratic fields. II”, Tokyo J. Math. 10:2 (1987), 259-270. MR 89g:11104 Zbl 0659.12008

[Bernstein 1976a] L. Bernstein, "Fundamental units and cycles. I", J. Number Theory 8:4 (1976), 446-491. MR 54 \#7427 Zbl 0352.10002

[Bernstein 1976b] L. Bernstein, "Fundamental units and cycles in the period of real quadratic number fields, I”, Pacific J. Math. 63:1 (1976), 37-61. MR 53 \#13166 Zbl 0335.10010

[Bernstein 1976c] L. Bernstein, "Fundamental units and cycles in the period of real quadratic number fields, II”, Pacific J. Math. 63:1 (1976), 63-78. MR 53 \#13167 Zbl 0335.10011

[Cohen and Lenstra 1984] H. Cohen and H. W. Lenstra, Jr., "Heuristics on class groups of number fields", pp. 33-62 in Number theory, Noordwijkerhout 1983 (Noordwijkerhout, 1983), Lecture Notes in Math. 1068, Springer, Berlin, 1984. MR 85j:11144 Zbl 0558.12002

[Dirichlet 1999] P. G. L. Dirichlet, Lectures on number theory, History of Mathematics 16, American Mathematical Society, Providence, RI, 1999. MR 2000e:01045 Zbl 0936.11004

[Hendy 1974] M. D. Hendy, "Applications of a continued fraction algorithm to some class number problems”, Math. Comp. 28 (1974), 267-277. MR 48 \#8440 Zbl 0275.12007

[Kaplansky 1998] I. Kaplansky, letter to Richard Mollin, Kenneth Williams and Hugh Williams, 23 November 1998. Available on the web page for this paper.

[Levesque and Rhin 1986] C. Levesque and G. Rhin, "A few classes of periodic continued fractions", Utilitas Math. 30 (1986), 79-107. MR 88b:11072 Zbl 0615.10014

[Levesque and Rhin 1991] C. Levesque and G. Rhin, "Two families of periodic Jacobi algorithms with period lengths going to infinity", J. Number Theory 37:2 (1991), 173-180. MR 92a:11119 Zbl 0723.11032

[Madden 2001] D. J. Madden, "Constructing families of long continued fractions", Pacific J. Math. 198:1 (2001), 123-147. MR 2002b:11014 Zbl 1062.11006

[Mendès France 1973] M. Mendès France, "Sur les fractions continues limitées", Acta Arith. 23 (1973), 207-215. MR 48 \#2083 Zbl 0228.10007

[Mollin and Cheng 2002] R. A. Mollin and K. Cheng, "Continued fractions beepers and Fibonacci numbers”, C. R. Math. Acad. Sci. Soc. R. Can. 24:3 (2002), 102-108. MR 2003e:11006 Zbl 1041. 11006

[Nyberg 1949] M. Nyberg, "Kulminerende og nesten-kulminerende kjedebrøker", Norsk Mat. Tidsskr. 31 (1949), 95-99. MR 11,329d Zbl 0040.30706

[Patterson 2003] R. D. Patterson, Creepers: Real quadratic fields with large class numbers, Ph.D. thesis, Macquarie University, Sydney, 2003. math.NT/0703519

[Perron 1950] O. Perron, Die Lehre von den Kettenbrüchen, 2nd ed., Chelsea, New York, 1950. MR 12,254b Zbl 0041.18206

[van der Poorten 1984] A. J. van der Poorten, "Some problems of recurrent interest", pp. 1265-1294 in Topics in classical number theory (Budapest, 1981), vol. 2, edited by G. Halasz, Colloq. Math. Soc. János Bolyai 34, North-Holland, Amsterdam, 1984. MR 86h:11015

[van der Poorten 1994] A. J. van der Poorten, "Explicit formulas for units in certain quadratic number fields", pp. 194-208 in Algorithmic number theory (Ithaca, NY, 1994), edited by L. M. Adleman and M.-D. Huang, Lecture Notes in Comput. Sci. 877, Springer, Berlin, 1994. MR 96b:11146 Zbl 0837.11004

[van der Poorten 1999] A. J. van der Poorten, "Beer and continued fractions with periodic periods", pp. 309-314 in Number theory (Ottawa, ON, 1996), edited by R. Gupta and K. S. Williams, CRM Proc. Lecture Notes 19, Amer. Math. Soc., Providence, RI, 1999. MR 2000e:11007 Zbl 0951.11025 
[van der Poorten 2002] A. J. van der Poorten, "Symmetry and folding of continued fractions", $J$. Théor. Nombres Bordeaux 14:2 (2002), 603-611. MR 2004k:11013 Zbl 1067.11001

[van der Poorten and Williams 1999] A. J. van der Poorten and H. C. Williams, "On certain continued fraction expansions of fixed period length”, Acta Arith. 89:1 (1999), 23-35. MR 2000m:11010 Zbl 0926.11005

[Schinzel 1960] A. Schinzel, "On some problems of the arithmetical theory of continued fractions", Acta Arith. 6 (1960), 393-413. MR 23 \#A3111 Zbl 0099.04003

[Schinzel 1961] A. Schinzel, "On some problems of the arithmetical theory of continued fractions, II”, Acta Arith. 7 (1961), 287-298. MR 25 \#2998 Zbl 0112.28001

[Shanks 1969] D. Shanks, “On Gauss's class number problems”, Math. Comp. 23 (1969), 151-163. MR 41 \#6814 Zbl 0177.07103

[Williams 1985] H. C. Williams, "A note on the period length of the continued fraction expansion of certain $\sqrt{D}$ ”, Utilitas Math. 28 (1985), 201-209. MR 87e:11016 Zbl 0586.10004

[Williams 1995] H. C. Williams, "Some generalizations of the $S_{n}$ sequence of Shanks", Acta Arith. 69:3 (1995), 199-215. MR 96a:11118 Zbl 0842.11004

[Williams and Buck 1994] K. S. Williams and N. Buck, "Comparison of the lengths of the continued fractions of $\sqrt{D}$ and $\frac{1}{2}(1+\sqrt{D})$ ", Proc. Amer. Math. Soc. 120:4 (1994), 995-1002. MR 94f:11062 Zbl 0794.11006

[Yamamoto 1971] Y. Yamamoto, "Real quadratic number fields with large fundamental units", Osaka J. Math. 8 (1971), 261-270. MR 45 \#5107 Zbl 0243.12001

Received November 23, 2004. Revised July 12, 2006.

ROGER D. PATTERSON

DEPARTMENT OF MATHEMATICS

MACQUARIE UNIVERSITY

SyDNEY, NEW SOUTH WALES

Australia

Current address:

DEPARTMENT OF MATHEMATICS AND STATISTICS

UNIVERSITY OF CALGARY

CAlgary, Alberta T2N 1N4

CANADA

rogerp@math.ucalgary.ca

Alfred J. VAN DER PoOrten

CENTRE FOR NUMBER THEORY RESEARCH

1 BIMBIL PL.

Killara, New South Wales 2071

Australia

alf@maths.usyd.edu.au

Hugh C. WiLliams

DePARTMENT OF MATHEMATICS AND StATISTICS

UNIVERSITY OF CALGARY

Calgary, Alberta T2N 1N4

CANADA

williams@math.ucalgary.ca 